OPEN ACCESS

Edited by:

Raffaella Balestrini,

Consiglio Nazionale delle Ricerche

(CNR), Italy

Reviewed by:

Luisa Lanfranco,

Università degli Studi di Torino, Italy

Cristiana Sbrana

Istituto di Biologia e Biotecnologia

Agraria (CNR), Italy

*Correspondence:

Vera Meyer

vera.meyer@tu-berlin.de

Specialty section:

This article was submitted to Fungi and Their Interactions,

a section of the journal

Frontiers in Microbiology

Received: 12 February 2018

Accepted: 16 April 2018

Published: 08 May 2018

Citation:

Fiedler MRM, Cairns TC, Koch O,

Kubisch C and Meyer V (2018) Conditional Expression of the Small

GTPase ArfA Impacts Secretion,

Morphology, Growth, and Actin Ring

Position in Aspergillus niger.

Front. Microbiol. 9:878.

doi: 10.3389/fmicb.2018.00878

\section{Conditional Expression of the Small GTPase ArfA Impacts Secretion, Morphology, Growth, and Actin Ring Position in Aspergillus niger}

\author{
Markus R. M. Fiedler, Timothy C. Cairns, Oliver Koch, Christin Kubisch and Vera Meyer*
}

Department of Applied and Molecular Microbiology, Institute of Biotechnology, Technische Universität Berlin, Berlin, Germany

In filamentous fungi, growth and protein secretion occurs predominantly at the tip of long, thread like cells termed hyphae. This requires coordinated regulation of multiple processes, including vesicle trafficking, exocytosis, and endocytosis, which are facilitated by a complex cytoskeletal apparatus. In this study, functional analyses of the small GTPase ArfA from Aspergillus niger demonstrate that this protein functionally complements the Saccharomyces cerevisiae ARF1/2, and that this protein is essential for A. niger. Loss-of-function and gain-of-function analyses demonstrate that titration of arfA expression impacts hyphal growth rate, hyphal tip morphology, and protein secretion. Moreover, localization of the endocytic machinery, visualized via fluorescent tagging of the actin ring, was found to be abnormal in ArfA under- and overexpressed conditions. Finally, we provide evidence that the major secreted protein GlaA localizes at septal junctions, indicating that secretion in A. niger may occur at these loci, and that this process is likely impacted by arfA expression levels. Taken together, our results demonstrate that ArfA fulfills multiple functions in the secretory pathway of $A$. niger.

Keywords: Aspergillus niger, tet-on, arf, protein secretion, glucoamylase, GTPase, actin, conditional gene expression

\section{INTRODUCTION}

Members of the fungal kingdom must acquire nutrients from their external environment to survive. An essential prerequisite for this saprophytic lifecycle is the ability to secrete enzymes for the breakdown of extracellular molecules. In filamentous species, secretion also is essential for growth of multicellular, tubular hyphae, whereby membrane components and enzymes necessary for cell wall synthesis are delivered to a continually extending tip.

This high secretion capacity has been harnessed in many biotechnological applications, and fungi such as Aspergillus niger are increasingly used as microbial cell factories in the pulp and paper, textile, detergent, beverage, food, agriculture, pharmaceutical, bio-fuel, and chemical industries (Meyer et al., 2016). Despite these utilities, our understanding of the molecular and cellular basis of filamentous growth, hyphal branching, and how these processes are spatially and temporally coupled with secretion, remains incomplete. This has led to constrains for the use of filamentous fungi in biotechnological applications. For example, several attempts to produce industrial relevant recombinant proteins in filamentous fungi led to production rates lower than the capacities published for homologous proteins (Grimm et al., 2005; Fiedler et al., 2013; Meyer et al., 2015). 
Elsewhere, targeted modification to transcription factors or chaperones for elevated secretion have been only partially successful (van Gemeren et al., 1998; Moralejo et al., 2001; Wiebe et al., 2001; Valkonen et al., 2003; Lombraña et al., 2004). Consequently, rational engineering of production strains with regulated developmental stages for optimal growth, expanded product portfolios, and enhanced secretion during industrial fermentation is currently not possible due to an incomplete understanding of growth and secretion.

With regards to fungal disease, which kills more people per year than malaria, and destroys enough crops to feed $\sim 10 \%$ of the world population annually (Cairns et al., 2016), a critical component of virulence for many fungal pathogens is invasive growth of polar hyphae into host tissues. This is often facilitated by secretion of hydrolytic enzymes for nutrient acquisition, or, most notably for plant infecting fungi, secretion of effector molecules which subvert, suppress, or manipulate host immunity to favor infection (Lo Presti et al., 2015). Consequently, a complete understanding of hyphal growth and secretion will enhance our understanding of the molecular basis of disease, and may lead to discovery of novel therapeutic targets.

The widely accepted model postulated by Taheri-Talesh in the model Aspergillus nidulans (Taheri-Talesh et al., 2008) states that polarized growth and secretion are coupled at the fungal tip. Vesicles packed with secretory proteins arise from the Golgi by budding (post-Golgi carrier and cargo; Luini et al., 2005), and travel along microtubules and actin filaments with the help of motor proteins to the apical dome, which is marked by cell end markers (Takeshita and Fischer, 2011; Takeshita et al., 2013; Ishitsuka et al., 2015), and enrich in a structure called the Spitzenkörper. Afterwards, they are transported toward the tip where they are tethered to the plasma membrane by a multi protein complex called the exocyst (Riquelme et al., 2014). Subsequent fusion is initiated by the interaction of vesicularsoluble $N$-ethylmaleimide-sensitive-factor attachment protein receptor (v-SNAREs) located within the vesicle membrane and target-SNAREs ( $t$-SNAREs) located at the plasma membrane. Next, the vesicle membrane and membrane bound proteins are incorporated into the plasma membrane, while the vesicular cargo is released into the external environment. While tip elongation proceeds through incorporation of delivered membrane and cell wall assembly via membrane bound enzymes, it is proposed that excess membrane and bound proteins are removed via endocytosis and cycled back to Golgi equivalents or the vacuole, which may ensure tip localization of cell end markers, and, consequently, polarized growth, and secretion (Ishitsuka et al., 2015). Endocytosis occurs at a collar, or ring, of actin patches and associated proteins, such as coronins (EchauriEspinosa et al., 2012), which is located $1-2 \mu \mathrm{m}$ behind the hyphal apex in A. nidulans (Taheri-Talesh et al., 2008). Although individual actin patches have an average lifespan lasting less than a minute, the position of the actin ring is tightly maintained (Taheri-Talesh et al., 2008). Thus, the position of the actin ring is likely critical for endocytosis, and ultimately filamentous growth. Characterizing key molecular components of this integrated system offers an opportunity to enhance our understanding of secretion and growth. Global gene expression analyses using either microarrays, or more recently, RNA-sequencing, offers an outstanding opportunity to understand growth and secretion at a systems level. In one such effort, Jörgensen et al. analyzed $A$. niger transcriptomes following carbon source dependent enhancement of protein secretion (Jørgensen et al., 2009). In this experiment, maltose and xylose were used as inducing and non-inducing conditions for secretion of the major A. niger extracellular protein glucoamylase, respectively. Elevated protein secretion resulted in transcriptional upregulation of over 90 genes encoding proteins which are known or predicted components of the $A$. niger secretory pathway, including glycosylation, protein folding, vesicular transport, and vacuolar sorting. Interestingly, this analysis demonstrated a gene predicted to encode an ADP ribosylation factor (An08g03690, ortholog of Saccharomyces cerevisiae Arf1/2) had 30\% elevated levels of expression following carbon-dependent enhancement of protein secretion. In addition, one predicted Arf activating protein (An11g02650, ortholog of S. cerevisiae Age2) and two predicted Arf guanine nucleotide exchange factors (An07g02190, ortholog of S. cerevisiae Sec7; An18g02490, ortholog of S. cerevisiae GEA2) were upregulated at a similar subtle level (10-30\%). This observation led us hypothesize that (i) the predicted ADP ribosylation factor encoded by An08g03690 is an important regulator of protein secretion in $A$. niger and that (ii) its subtle level of upregulation is critical for ensuring high level secretion.

ADP ribosylation factors of the Arf/Sar family are small GTPase proteins that regulate a diverse range of processes that have been well described in the budding yeast S. cerevisiae, including vesicle formation and trafficking, cytoskeletal rearrangements, cell polarity, and budding (Roth, 1999; Lambert et al., 2007; Suda et al., 2018). The family consists of seven members (Arf1, Arf2, Arf3, Arl1, Arl3, Cin4, and Sar1). Arf1 and Arf2 are redundant, and together with Arl1 are major regulators of the secretory pathway, where they control consecutive steps from ER-Golgi, Golgi-ER, Golgi-plasma membrane and endosomes-trans-Golgi. Arf1/2 play a critical role in formation of vesicle coats at distinct steps in intracellular vesicle trafficking in the Golgi, specifically formation of COPI vesicles and clathrin coated vesicles at cis and trans Golgi cisternae, respectively (Roth, 1999; Suda et al., 2018).

Arf proteins, like all small GTPase proteins, cycle between a GTP-bound (active) and GDP-bound (inactive) forms. In their GDP-bound form, small GTPases are distributed within the cytoplasm, but can be targeted toward a specific membrane by their N-terminal myristoylation group binding phospholipids (Franco et al., 1996). Subsequently, GTP exchange factors (GEF) promote the exchange of GDP with GTP, upon which Arf proteins are activated. The GEFs for Arf1 in yeast have been comprehensively identified, and include Geal/2 and Sec7 (reviewed in Roth, 1999; Suda et al., 2018). GTPase activating proteins (GAP) catalyze the hydrolysis of bound GTP to GDP, thereby inactivating the GTPase, which diffuses back into the cytoplasm. In S. cerevisiae, for example, cognate GAPs for Arf1 include Glo3, Age1/2, and Gcs1 (Poon et al., 1996, 1999; Zhang et al., 1998; Suda et al., 2018). In addition to the well characterized role of Arfs in budding yeast, these GTPases have also been well studied in mammals (Gillingham and Munro, 2007), and 
there is growing interest in the role of these proteins regulating morphology and virulence during disease, for example in the human infecting dimorphic yeast Candida albicans and the zygomycete Mucor circinelloides (Labbaoui et al., 2017; PatiñoMedina et al., 2017). Despite comprehensive molecular and genetic characterization of Arfs, their cognate GEFs and GAPs, and the regulatory role of these proteins on multiple processes in yeast and mammalian systems, little is known about their role in Aspergilli, and filamentous fungi in general. A single study has demonstrated that an orthologue of S. cerevisiae Arf1 and Arf2 localized to Golgi in A. nidulans (Lee and Shaw, 2008). Additionally, these authors were unable to recover disruption mutants for $A$. nidulans $A R F 1 / 2$ orthologue, indicating it was essential. Moreover, over-expression of ArfA impacted cell polarity. However, comprehensive functional analyses of this gene and the encoded proteins have not been conducted in an Aspergillus spp.

In this work we provide the first functional analysis of ArfA in A. niger, and identify a key role of this protein in regulating growth, morphology, and secretion. Conditional expression following replacement of the native $\operatorname{arfA}$ promoter with the regulatable Tet-on system (Meyer et al., 2011) demonstrated that ArfA is essential. Subsequent loss-of-function and gainof-function analyses demonstrate that ArfA impacts hyphal growth rate, hyphal tip morphology, and protein secretion. Subcellular localization experiments of fluorescently labeled proteins associated with cytoskeletal elements provide evidence that the position of the endocytic actin ring is impacted by both lowered and elevated levels of $\operatorname{arf} A$ expression. We also provide new evidence that secretion in A. niger may occur at septa.

\section{MATERIALS AND METHODS}

\section{Strains, Growth Conditions, and Molecular Techniques}

A. niger and $S$. cerevisiae strains used in this study are listed in Table 1. Strains of $A$. niger were grown at $30^{\circ} \mathrm{C}$ in minimal medium (MM) (Meyer et al., 2010) or complete medium (CM), consisting of minimal medium (MM) supplemented with $1 \%$ yeast extract and $0.5 \%$ casamino acids. $100 \mu \mathrm{g} / \mathrm{ml}$ of hygromycin, $10 \mathrm{mM}$ uridine, or $10 \mathrm{mM}$ histidine were added to the medium when required. Yeast strains were cultivated at $28^{\circ} \mathrm{C}$ in YPG (1\% yeast extract, $2 \%$ peptone, $2 \%$ glucose) or YNB (Formedium, UK) supplemented with $2 \%$ glucose, $20 \mathrm{mg} / \mathrm{l}$ leucine, histidine, uridine, and methionine when needed. FOA counterselection of A. niger strains was performed as described earlier (Arentshorst et al., 2012). S. cerevisiae counterselection was performed on YNB + FOA plates (Boeke et al., 1984) supplemented with $50 \mathrm{mg} / \mathrm{l}$ histidine, $50 \mathrm{mg} / \mathrm{l}$ methionine, and $100 \mathrm{mg} / \mathrm{l}$ leucine. Strains were grown at $28^{\circ} \mathrm{C}$ for $3-5$ days.

All molecular techniques were performed according to standard procedures (Green and Sambrook, 2012). Plasmids were constructed using Gibson assembly (Gibson, 2011) and the transformation, genomic DNA extraction and Southern blot were performed as described elsewhere (Meyer et al., 2010).
Protein production in shake flasks was performed as follows: $5 \times 10^{6}$ conidia/ml were inoculated in $20 \mathrm{ml} \mathrm{MM} \mathrm{medium}$ supplemented with $5 \%$ glucose and different concentrations of doxycycline in $100 \mathrm{ml}$ Erlenmeyer flasks, and cultivated at $30^{\circ} \mathrm{C}$ and $250 \mathrm{rpm}$ on a horizontal shaker for $72 \mathrm{~h}$. One milliliter samples for microscopic analysis were taken, the biomass was harvested by filtration, washed once with physiological salt solution and supernatants were collected for further analysis.

\section{Construction of a PgpdA::glaA $514:: d t o m a t o$ Construct}

PgpdA::glaA ${ }_{514}::$ dtomato expression construct (pMF30.1) was constructed by amplification of gla $A_{514}$ and dtomato via PCR (Supplementary Table S1). For dtomato, plasmid pBN018-60-61 (van Munster et al., 2013) was used as template and the short version of the glucoamylase gene $g l a A_{514}$ was amplified from genomic DNA of N402. Both fragments were cloned into the EcoRI and PmeI opened plasmid pVG2.2 (Meyer et al., 2011) via Gibson assembly (Gibson, 2011).

\section{Construction of a hisB Disruption and Tet-On::arfA::hisB Expression Construct}

The construction of the hisB disruption construct is described elsewhere (Fiedler et al., 2017). The Tet-On::arfA::hisB expression plasmid (pFH1.3) was created by inserting arfA amplified via PCR (Supplementary Table S1) from the genomic DNA of N402 into the unique PmeI restriction site of Tet-On::hisB (pTG1.2 Fiedler et al., 2017) via Gibson assembly.

\section{Construction of a arfA::hygR Deletion Cassette}

The $5^{\prime}$ and $3^{\prime}$ regions of $\operatorname{arf} A$ and the hygromycin resistance gene were amplified in a bipartite approach as published recently (Arentshorst et al., 2015) using primers listed in Supplementary Table S1 and cloned into pJET1.2 (Thermo, USA) via Gibson assembly giving rise to bipartite containing plasmids pCK1.1 (ParfA-hygR) and pCK2.6 (hygR::TarfA), respectively. Both plasmids were linearized with DraI giving rise to two bipartites with a length of $3.1 \mathrm{~kb}$ each.

\section{Construction of a}

\section{arfA::dtomato::hygR::TarfA Cassette}

C-terminal ArfA labeling was chosen because of an N-terminal myristoylation site which was shown to be essential for proper localization (Liu et al., 2009). The promoter sequence of $\operatorname{arfA}$, dtomato-TtrpC and the hygromycin resistance gene were amplified using primers listed in Supplementary Table S1 from genomic DNA of N402, pMF30.1, or pAN7.1(Punt et al., 1987) using PCR and ligated into the XhoI and NdeI opened plasmid pCK2.6 via Gibson assembly giving rise to pMF44.6.

\section{Microscopy}

Different cultivation techniques were applied for microscopy. Cultivation for cLSM microscopy of $A$. niger was performed as described recently (Kwon et al., 2014). In brief, spores were spotted in MM plates, supplemented with different concentrations of doxycycline when needed and incubated at 
TABLE 1 | A. niger strains used in this work.

\begin{tabular}{|c|c|c|}
\hline Name & Genotype & References \\
\hline N402 & $\operatorname{csp} A 1$ & Bos et al., 1988 \\
\hline MA70.15 & kusA::amdS, pyrG- & Meyer et al., 2007 \\
\hline FG7 & $\Delta k u s A$, pyrG ${ }^{+}$, egfp::sncA-TsncA-AopyrG-TsncA (derivative of MA70.15) & Kwon et al., 2014 \\
\hline MF9.1 & $\Delta k u s A, p y r G^{-}$, egfp::sncA, $\Delta$ glaA, pyrG ${ }^{-}$(derivative of MF7.4) & Fiedler, 2017 \\
\hline MF31.2 & $\Delta k u s A$, pyrG ${ }^{+}$, egfp::sncA, $\Delta$ glaA, PgpdA::glaA 514 -dtomato (derivative of MF9.1) & This study \\
\hline MF26.1 & $\Delta$ kusA, pyrG+ ${ }^{+}$egfp::sncA, $\Delta$ glaA, hisB::DR-AopyrG-DR (derivative of MF9.1) & This study \\
\hline MF27.1 & $\Delta k u s A$, pyrG ${ }^{-}$, egfp::sncA, $\Delta$ glaA, pyrG ${ }^{-}$, hisB $^{-}$(derivative of M26.1) & This study \\
\hline MF32.6 & 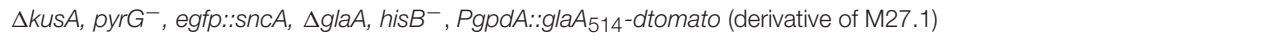 & This study \\
\hline $\mathrm{FH} 1.1$ & $\Delta k u s A$, pyrG ${ }^{+}$, egfp::sncA, $\Delta$ glaA, hisB::Tet-on-arfA, PgpdA::glaA 514 -dtomato (derivative of M32.6) & This study \\
\hline MF45.5 & $\Delta$ kusA, pyrG ${ }^{+}$, egfp::sncA, $\Delta g l a A$, hisB::Tet-on-arfA, PgpdA::glaA 514 -dtomato, $\Delta$ arfA::hygR (derivative of FH1.1) & This study \\
\hline MF47.11 & $\Delta k u s A$, pyrG ${ }^{+}$, egfp::sncA, ParfA::arfA::dtomato:hygR (derivative of FG7) & This study \\
\hline MA141.1 & $\Delta$ kusA, pyrG ${ }^{+}$, PgpdA::glaA::sGFP::HDEL::TtrpC-pyrG (derivative of MA70.15) & Carvalho et al., 2011 \\
\hline MF48.11 & $\Delta$ kusA, pyrG ${ }^{+}$, PgpdA::glaA::sGFP::HDEL::TtrpC-pyrG, ParfA::arfA::dtomato:hygR (derivative of MA141.1) & This study \\
\hline Ren1.10 & $\Delta$ kusA, pyrG ${ }^{+}$, PgmtA::eYFP::gmtA::TgmtA (derivative of MA70.15) & Carvalho et al., 2011 \\
\hline MF49.1 & $\Delta$ kusA, pyrG ${ }^{+}$, PgmtA::eYFP::gmtA::TgmtA, ParfA::arfA::dtomato:hygR (derivative of Ren1.10) & This study \\
\hline MK6.1 & $\Delta k u s A, a b p A:: e c f p$ (derivative of MA70.15) & Kwon et al., 2013 \\
\hline MF46.1 & $\Delta k u s A$, pyrG ${ }^{-}$, egfp::sncA, $\Delta$ glaA, hisB::Tet-on-arfA, $\triangle$ arfA::hygR (derivative of MF45.5) & This study \\
\hline MF58.5 & $\Delta$ kusA, pyrG ${ }^{+}$, egfp::sncA, $\Delta g l a A$, hisB::Tet-on-arfA, $\Delta$ arfA::hygR, abpA::ecfp (derivative of MF46.1) & This study \\
\hline BY4742 & MAT $\alpha$ his $3 \Delta 1$ leu2 $\Delta 0$ lys $2 \Delta 0$ ura3 $\Delta 0$ & Baker Brachmann et al., 1998 \\
\hline 3890 & MATa, his3 $\Delta 1$, leu2 $\Delta 0$, met15 $\Delta 0$, ura3 $\Delta 0$, ydl192w::kanMX & Winzeler et al., 1999 \\
\hline MF52.2 & MATa, his3 $\Delta 1$, leu2 $\Delta 0$, met15 $\Delta 0$, ydl192w::kanMX, pRS416::ARF1::URA3 (derivative of 3890) & This study \\
\hline MF59.1 & MATa, his3 $\Delta 1$, leu2 $\Delta 0$, ydl192w::kanMX, pRS416::ARF1::URA3, arf2::MET15 (derivative of MF52.2) & This study \\
\hline MF61.1 & MATa, his3 $\Delta 1$, leu2 $\Delta 0$, ydl192w::kanMX, pRS416::ARF1::URA3, arf2::MET15, pRS315PGI-ARF1 (derivative of MF59.1) & This study \\
\hline MF62.1 & MATa, his3 $\Delta 1$, leu2 $\Delta 0$, ydl192w::kanMX, pRS416::ARF1::URA3, arf2::MET15, pRS315PGl-arfA (derivative of MF59.1) & This study \\
\hline CK5.1 & MATa, his3 $\Delta 1$, leu2 $\Delta 0$, ydl192w::kanMX, pRS416::ARF1::URA3, arf2::MET15, pRS315PGI (derivative of MF59.1) & This study \\
\hline
\end{tabular}

$22^{\circ} \mathrm{C}$ for 2 days, following cutting out of the colony and placing it upside down into a glass bottom petri dish (Kwon et al., 2014). Liquid MM medium (supplemented with the same concentration of doxycycline which was present in the MM plate) was added and cells were incubated at $22^{\circ} \mathrm{C}$ until they resumed growth. Fluorescence and DIC images were taken using an inverted TCS SP8 (Leica, Germany). For fluorescence microscopy, cultivation was performed as described earlier (Fiedler et al., 2014). In brief, cover slides were placed on the bottom of a Petri dish containing $5 \mathrm{ml}$ MM medium (supplemented with $100 \mathrm{mM}$ MES pH6.5 and different concentrations of doxycycline when needed), inoculated with $1 \times 10^{5}$ spores $/ \mathrm{ml}$ and cultivated at $28^{\circ} \mathrm{C}$ for $12 \mathrm{~h}$. Images were acquired using a DMI6000 fluorescence microscope (Leica, Germany).

\section{Growth-Plate Assays}

Defined spore titers of $A$. niger strains were spotted on MM medium supplemented with different additives and varying doxycycline concentrations in biological triplicate and incubated at $30^{\circ} \mathrm{C}$ for 3 days, after which representative images were captured.

\section{Glucoamylase Analysis}

Western analysis was performed according to standard procedures (Green and Sambrook, 2012) and as described earlier (Punt et al., 1998). Briefly, a monoclonal anti-glucoamylase antibody produced in mouse (kindly provided by Prof. Peter Punt, University of Leiden) was used as a primary antibody. The primary antibody was detected with an anti-mouse-HRP conjugated antibody (Agilent Technologies, USA). Primary and secondary antibody incubations were performed in PBS-T (137 mM NaCl, $2.7 \mathrm{mM} \mathrm{KCl,} 1 \mathrm{mM} \mathrm{Na} 2 \mathrm{HPO}_{4}, 0.2 \mathrm{mM} \mathrm{KH}_{2} \mathrm{PO}_{4}$, $0.1 \%$ Tween-20) supplemented with $5 \%$ dry milk. The primary antibody incubation was performed at $4{ }^{\circ} \mathrm{C}$ for $16 \mathrm{~h}$, after which the blot was incubated with the secondary antibody at room temperature for $1 \mathrm{~h}$. Chemiluminescence reaction was performed by using an ECL Prime Western Blotting Detection Kit (GE Healthcare). Band intensities were analyzed using the open source program Image J and the signal intensity was normalized against the corresponding biomass and subsequently against the signal intensity of the corresponding control strain.

\section{Construction of the S. cerevisiae complementation strains}

To construct $S$. cerevisiae strains which were used for complementation analysis, we followed an approach which was published earlier (Takeuchi et al., 2002). In brief, the S. cerevisiae ydl192w::kanMX (3890) strain from the Saccharomyces Genome Deletion Project (Winzeler et al., 1999) lacking a functional copy of $A R F 1$ was transformed with the ectopically replicating plasmid pMF46.3 derived from pRS416 (purchased from Stratagene) giving rise to MF52.2. pMF46.3 carries the full length $A R F 1$ 
gene flanked by $471 \mathrm{bp}$ of the $5^{\prime}$ and $833 \mathrm{bp}$ of the $3^{\prime}$ region of ARF1 amplified from the genome of BY4742 using primers listed in Supplementary Table S1 ligated into pRS416 using unique BamHI and EcoRI restriction sites. Subsequently, an ARF2::MET15 deletion cassette was amplified from the genome of BY4742 using primers listed in Supplementary Table S1 and transformed into MF52.2 to create MF59.1. Finally GPD driven expression plasmids for ARF1 (pMF48.1) or $\operatorname{arfA}$ (pMF49.1) were constructed via inserting the respective genes amplified via PCR from the genome of BY4742 and N402, respectively into a unique BamHI restriction site of the ectopically replicating expression plasmid pRS315-PGI (kindly provided by Dr. T. H. Chang, Ohio State University, Weaver et al., 1997) carrying LEU2 as selection marker and subsequent transformation into MF59.1 to generate MF61.1 (ARF1), MF62.1 (arfA), or CK5.1 (plasmid control).

\section{RESULTS}

\section{The A. niger ArfA Encoding Gene Functionally Complements $S$. cerevisiae ARF1/2}

Microarray analyses of $A$. niger gene expression during carbon source dependent upregulation of secretion has identified transcriptional deployment of numerous genes, including those predicted to function in protein folding, $\mathrm{N}$-glycosylation, vesicle packaging, and ER to Golgi transport (Jørgensen et al., 2009). Interestingly, these analyses also identified upregulated expression of gene An08g03690, which is predicted to encode an Arf protein, during elevated secretion. Subsequent BLAST analyses of the encoded amino acid sequence revealed An08g03690 has sequence homology of $76.1 \%$ when compared with S. cerevisiae Arf1 (Supplementary Table S2). Further BLAST searches of the An08g03690 protein sequence revealed 97.8\% sequence homology to the A. nidulans ArfA (Lee and Shaw, 2008, data not shown). We consequently named the protein encoded by the An08g03690 gene in A. niger ArfA. In order to assess whether the An08g03690 gene encodes an ADP ribosylation factor, we conducted a $S$. cerevisiae complementation assay comparable to those previously described (Takeuchi et al., 2002). We firstly generated S. cerevisiae strain MF59.1, which expresses an episomal plasmid constitutively expressing a functional $A R F 1$ gene that can be removed via 5 -fluororotic acid (FOA) counterselection (Figure 1). This isolate is also deleted for genes YDL192W and YDL137W, which encode proteins Arf1 and Arf2, respectively. Given that deletion of both paralogues is lethal in S. cerevisiae, FOA counterselection in isolate MF59.1 must be complemented with supply of a functional ARF for cell survival. Next, an expression construct containing the A. niger arfA gene (An08g03690) was transformed into MF59.1, giving isolate MF62.1. Additionally, plasmids encoding the endogenous $S$. cerevisiae $A R F 1$, and an unmodified vector control, were also introduced to MF59.1, to give strains MF61.1 and CK5.1, respectively. These isolates were used as positive and negative controls for functional ARF complementation in the subsequent assay. All three strains were able to grow on YNB supplemented with histidine (Figure 1). Plating the three strains on YNB
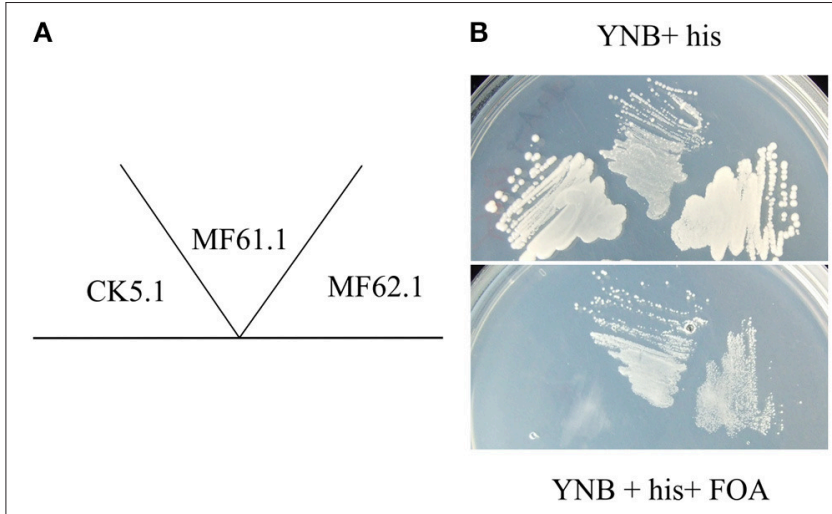

FIGURE 1 | A. niger ArfA functionally complements S. cerevisiae Arf1/2. The S. cerevisiae ARF1/2 double null, containing an FOA counterselectable ARF1 expression plasmid, was transformed with vectors expressing the native S. cerevisiae ARF1 (MF61.1), A. niger arfA (MF62.1), or empty vector control (CK5.1). These strains were grown on agar plates as depicted schematically (A). Media consisted of yeast nitrogen base (YNB) supplemented with $10 \mathrm{mM}$ histidine (his, upper panel), which was supplemented with FOA (lower panel). CK5.1 was unable to grow following counterselection, whereas both isolates expressing the $S$. cerevisiae ARF1 or A. niger arfA demonstrated comparable growth, (B) indicating $A$. niger arfA functionally complements $A R F 1 / 2$.

supplemented with histidine and FOA revealed no growth for the negative control (CK5.1), but normal growth for the A. niger $\operatorname{arfA}$ (MF62.1) and endogenous ARF1 (MF61.1) complemented strains (Figure 1). This assay confirmed that the $A$. niger arfA is able to complement loss of $A R F 1 / 2$ in $S$. cerevisiae and also provides indirect evidence that the protein encoded by this gene functions is an ADP ribosylation factor.

\section{Gene Functional Analysis Demonstrates arfA Is Essential in A. niger, and Is Required for Normal Growth and Resistance to Secretion Stress}

As deletion of both paralogues ARF1/2 in S. cerevisiae, and arfA in A. nidulans is lethal (Stearns et al., 1990; Lee and Shaw, 2008), we reasoned that the gene might be essential in $A$. niger. In support of this hypothesis, 4 independent transformations did not result in successful purification of a homokaryotic deletion strain. Indeed, only heterokaryons were rescued from primary transformation plates, and subcultured spores were unable to grow on MM supplemented with hygromycin (data not shown). In order to assess the arfA loss-of-function phenotype of in $A$. niger, we placed the arfA gene under control of the doxycycline inducible Tet-on system (Meyer et al., 2011) integrated into the hisB locus (Fiedler et al., 2017), to generate isolate FH1.1. The Tet-on conditional expression system is titratable in A. niger, and enables accurate modifications of gene expression by addition of the highly stable tetracycline derivative doxycycline to growth media (Meyer et al., 2011; Helmschrott et al., 2013; Wanka et al., 2016). For isolate FH1.1, which contains the endogenous $\operatorname{arfA}$ gene, any addition of doxycycline to growth media results in $\operatorname{arfA}$ overexpression. For loss-of-function analyses, 
strain MF45.5 was generated by deletion of the native arfA gene in isolate FH1.1. In order to assess protein secretion in subsequent microscopic and Western blot experiments, both isolates also constitutively expressed a C-terminal dtomato tagged glucoamylase gene glaA, under control of the constitutive A. niger glyceraldehyde-3-phosphate dehydrogenase promoter at the pyrG locus (Table 1). Consequently, for growth assays, we used isolate MF31.2 as a control, which also constitutively expresses a C-terminally dtomato tagged glaA, but has no modifications to arfA expression (Table 1). Titration of arfA gene expression by addition of various concentrations of doxycycline to growth media allowed us thus to study loss-offunction and gain-of-function phenotypes in the same isolate. As shown in Figure 2, the doxycycline controllable arfA expression strain MF45.5 was unable to grow on plates lacking doxycycline, while addition of $0.2 \mu \mathrm{g} / \mathrm{ml}$ of doxycycline led to an intermediate phenotype showing reduced growth and sporulation in comparison to the wildtype progenitor strain. These data demonstrate that arfA is indeed essential in A. niger. With increased doxycycline concentration $(0.3$ and $1 \mu \mathrm{g} / \mathrm{ml})$, wildtype-like growth phenotype was restored in MF45.5. Growth and sporulation of $A$. niger, however, were significantly reduced when $\operatorname{arfA}$ was overexpressed at $10 \mu \mathrm{g} / \mathrm{ml}$ doxycycline for both arfA expression strains (MF45.5 and FH1.1), demonstrating not only a clear overexpression phenotype but also suggesting that native $\operatorname{arfA}$ expression itself is under delicate control to ensure proper growth. In order to test whether the observed growth defects in $\operatorname{arfA}$ conditional expression isolates were due to misregulation of the secretory pathway, we conducted phenotypic screens of these mutants under conditions known to affect or perturb fungal secretion, including growth on starch as a sole carbon source, which requires secretion of amylolytic proteins for external substrate digestion. Growth of isolate MF45.5 was strongly impaired compared to wild-type when grown on starch media supplemented with $0.2-0.3 \mu \mathrm{g} / \mathrm{ml}$ doxycycline, suggesting a defect in secretion (Figure 2). Interestingly, overexpression of $\operatorname{arfA}$ also resulted in sensitivity to secretion stress in isolate MF45.5. We also tested growth on MM supplemented with calcium, which is a central regulator of the secretory pathway of filamentous fungi and yeasts (Sambrook, 1990; Lew, 2011; Liu, 2012; Figure 2). Strain FH1.1, which has an endogenous arfA copy and a Tet-on regulated allele did not display sensitivity to secretion stress at low doxycycline concentrations $(0-0.3 \mu \mathrm{g} / \mathrm{ml})$, yet at higher concentrations $(1-10 \mu \mathrm{g} / \mathrm{ml})$ demonstrated reduced growth relative to the control (Figure 2), further suggesting that overexpression of arfA results in sensitivity to secretion stress.

Given that the secretory pathway is essential for transporting cell wall synthesizing enzymes and material toward apical regions during hyphal growth, we reasoned that loss-of-function and gain-of-function arfA mutants would also be sensitive to cell wall interfering compounds such as calcofluor white (CFW) and congo red (CR), which are known inhibitors of chitin and glucan assembly, respectively (Roncero and Durán, 1985; Ram and Klis, 2006; He et al., 2014). Growth of strain MF45.5 was indeed stronger impaired than the respective control strains FH1.1. and MF31.1 on plates containing CFW and CR (Figure 2), strongly supporting the hypothesis of defective delivery of cell wall components in $\operatorname{arfA}$ loss and gain-of-function strains. We finally challenged the strains with sorbitol, which causes osmotic stress at both the plasma membrane and cell wall. Growth in strain MF45.5 under decreased $\operatorname{arfA}$ expression was comparable to that of the progenitor strain, and minor defects in sporulation were observed under native or overexpression conditions (Figure 2). Taken together, these data suggest that ArfA plays essential roles regulating the secretion pathway in A. niger.

\section{Titration of arfA Expression Impacts A. niger Secretion and Colony Macromorphology in Submerged Culture}

We hypothesized that $\operatorname{arfA}$ downregulation and overexpression would affect the total protein secretion capacity of $A$. niger. We therefore measured secretion of fluorescently tagged glucoamylase GlaA, one of the major secreted proteins in A. niger, in addition to total extracellular protein, after $72 \mathrm{~h}$ growth in shake flask culture (Figure 3 and Supplementary Figure S1). We also measured biomass and pellet diameter to assess macromorphological changes following arfA conditional expression (Figure 3 and Supplementary Figure S2). We detected minor but statistically significant modifications to biomass in arfA loss- and gain-of-function mutants at 0.5 and $10 \mu \mathrm{g} / \mathrm{ml}$ doxycycline respectively (Figure $3 \mathbf{A}$ ). Interestingly, arfA overexpression resulted in reduced pellet diameter (Figure 3B), which may be favorable for industrial fermentation applications (Wucherpfennig et al., 2012). Additionally, total protein secretion was increased in ArfA overexpression isolate FH1.1 under all conditions (Figure 3C). This, however, cannot be explained exclusively by changes in pellet diameter, as secretion was also elevated in FH1.1 at $0.25 \mu \mathrm{g} / \mathrm{ml}$ doxycycline, when $A$. niger macromorphology was comparable to control strains (Figure 3B). Extracellular GlaA (Figure 3D) was elevated in FH1.1 $(10 \mu \mathrm{g} / \mathrm{ml}$ doxycycline) suggesting that higher levels of arfA expression results in increased secretion of this protein (Figure 3D). It should be noted that in contrast to isolate FH1.1, strain MF45.5 demonstrated increased GlaA secretion exclusively at $0.5 \mu \mathrm{g} / \mathrm{ml}$ doxycycline, which could be due to changes in pellet diameter between these isolates at various doxycycline concentrations (Figure 3B).

With regards to analyses of arfA downregulation using $0.25 \mu \mathrm{g} / \mathrm{ml}$ doxycycline in mutant MF45.5, colony macromorphology was comparable, yet GlaA production was reduced relative to the MF32.1 control (Figure 3D). Interestingly, total protein secretion in MF45.5 during downregulation of arfA was also comparable (Figure 3C). These data suggest that lower arfA levels might impact a GlaA-specific component of the secretory system, but does not detectably impact the total secretion capacity in A. niger. In summary, our data indicate that (i) overexpression of the ArfA encoding gene elevates secretion of total protein and GlaA, while concomitantly reducing pellet diameter and (ii) lower ArfA levels causes a reduced secretion of GlaA but not total protein. 


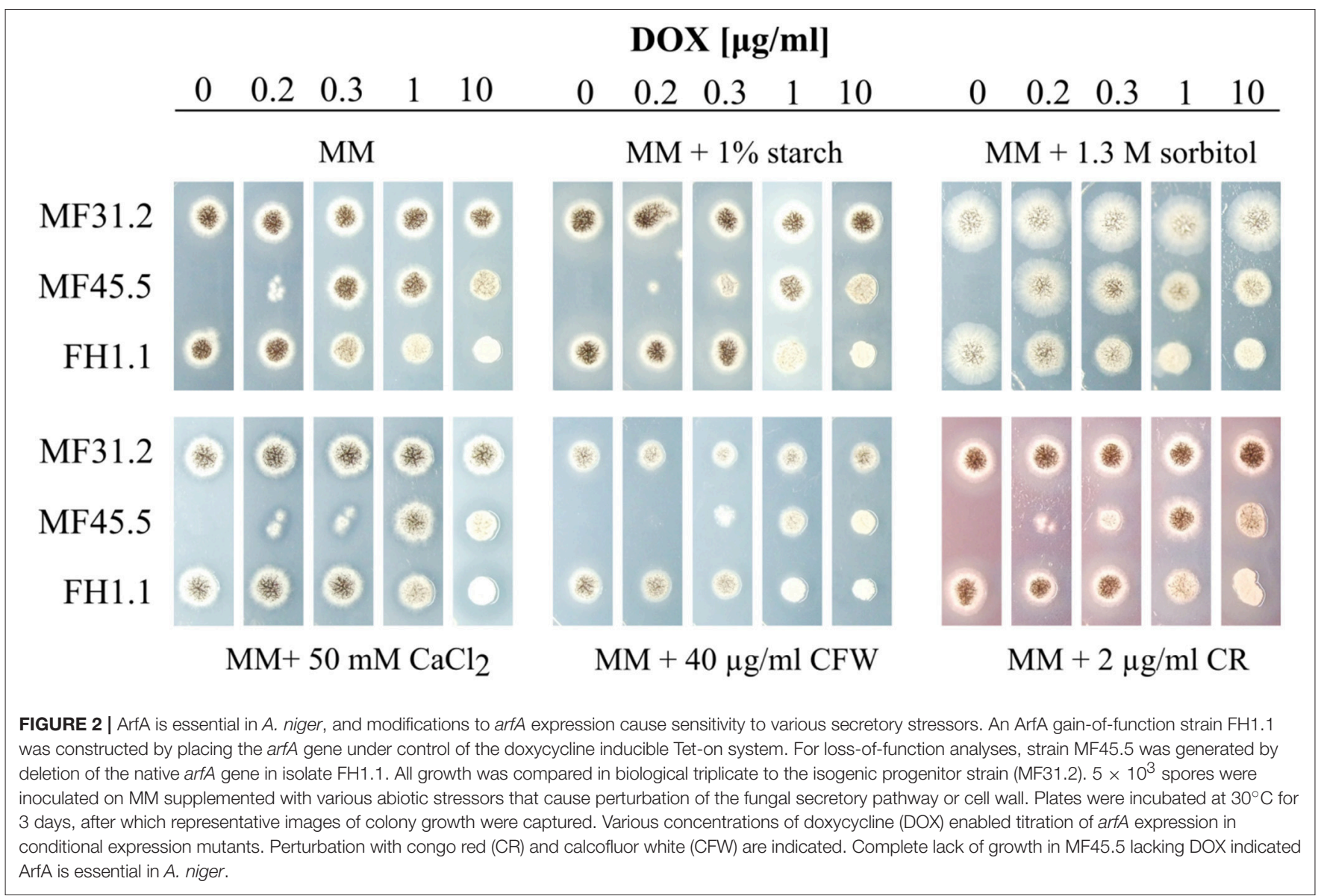

\section{Reduced arfA Expression Results in Hyphal Bursting at Intercalary Regions}

In order to further interrogate modifications in secretion and colony macromorphology following titration of arfA expression, we concomitantly monitored hyphal morphology and GlaA localization (Figure 4), in addition to quantitative assessment of growth rates (Figure 5) for strains MF31.2, MF45.5 and FH1.1. Addition of $1 \mu \mathrm{g} / \mathrm{ml}$ doxycycline resulted in wildtypelike morphology (Figure 4) and growth rates (Figure 5) for all tested isolates. However, intracellular localization of the reporter protein glucoamylase was still observed for some hyphae of isolate MF45.5 (Figure 4).

Following addition of $0.25 \mu \mathrm{g} / \mathrm{ml}$ of doxycycline to growth media, isolates MF31.2 and FH1.1 demonstrated comparable growth rates, hyphal morphology, and absence of intracellular GlaA. However, loss-of-function isolate MF45.5 resulted in abnormal hyphal branching, curved growth, and swelling (Figure 4) and reduced growth rate (Figure 5). This was surprising, given that biomass was comparable between MF45.5 and the progenitor control in shake flask culture (Figure 3A). We hypothesize that the most likely explanation of the discrepancies between hyphal growth rates on solid media (Figure 5) and biomass in shake flask culture (Figure 3A) are related to the different growth parameters between the assays, which were $22^{\circ} \mathrm{C}$ for $28 \mathrm{~h}$ and $30^{\circ} \mathrm{C}$ for $72 \mathrm{~h}$, respectively. These data suggest that ArfA regulation in A. niger is partially dependent on temperature, and/or a solid growth substrate.

Additionally, under loss-of-function conditions using $0.25 \mu \mathrm{g} / \mathrm{ml}$, isolate MF45.5 demonstrated significant punctate localisation of intracellular GlaA, with numerous hyphae demonstrating rupture of hyphal compartments (Figure 4). This bursting was followed by release of GlaA into the surrounding medium (Figure 4). It is notable that from over 100 hyphae analyzed, cellular burst occurred mostly at intercalary regions (29\%), whereas bursting of hyphae at the tip was considerably less frequent (5\%, Figure 4). Hyphal bursting may contribute to decreased GlaA secretion in loss-of-function isolate MF45.5 during submerged growth due to cell death from intercalary rupture (Figure 3D).

Interestingly, when $\operatorname{arfA}$ was overexpressed using $10 \mu \mathrm{g} / \mathrm{ml}$ doxycycline in isolates MF45.4 and FH1.1, intracellular GlaA was not observed, and morphology was comparable to the control isolate (Figure 4). However, growth rates were impaired relative to MF31.2 for both gain-of-function strains (Figure 5). This latter observation likely explains significant changes in colony macromorphology in submerged culture (Figure 3B). Taken together, these data further support a critical role for ArfA in regulating growth, morphology, and secretion in $A$. niger. 

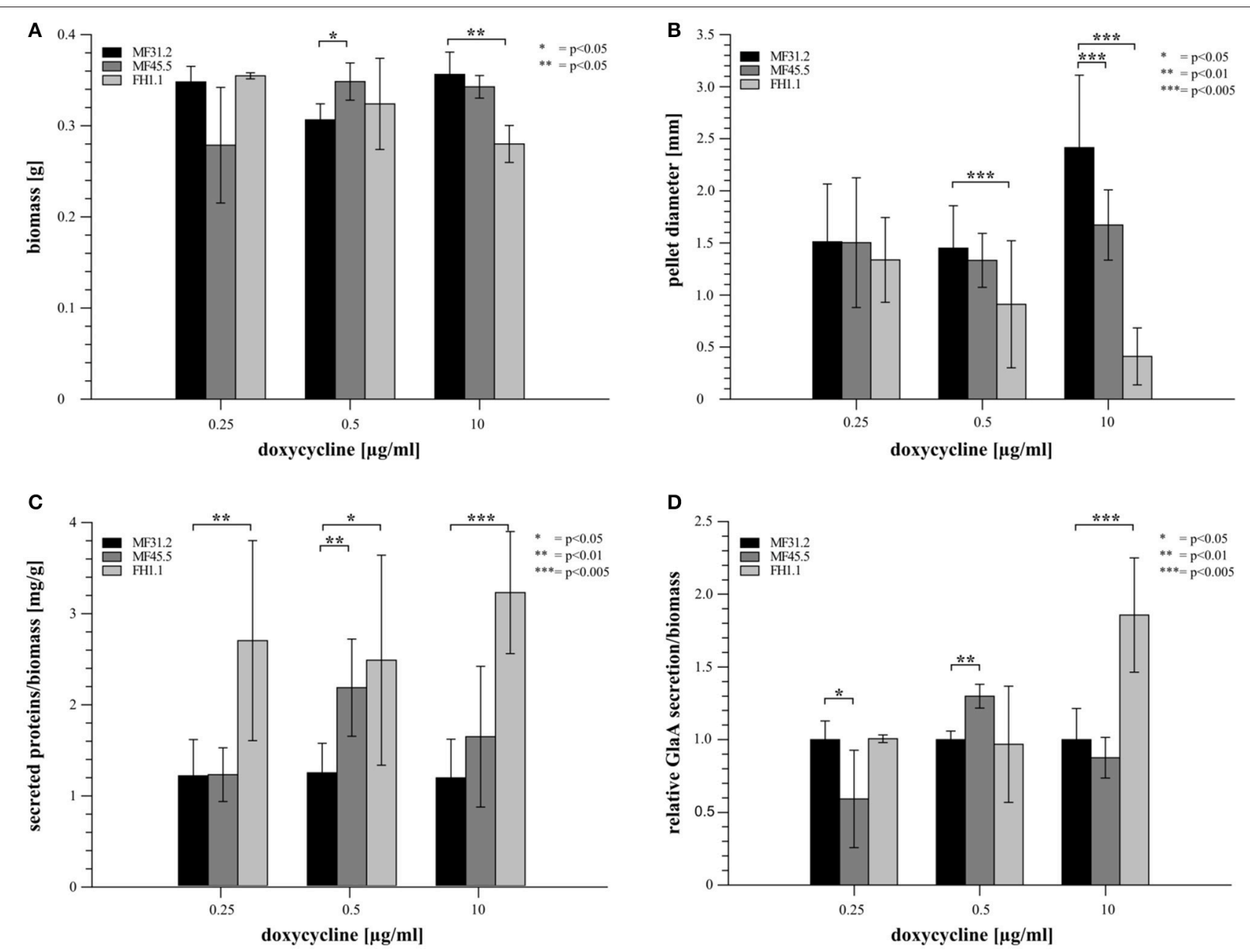

FIGURE 3 | Modification of arfA expression impacts colony macromorphology and protein secretion in submerged cultures. $20 \mathrm{ml}$ of MM supplemented with $5 \%$ glucose and various doxycycline concentrations $\left(0.25,0.5\right.$, and $10 \mu \mathrm{g} / \mathrm{ml}$ ) were inoculated with a final concentration of $5 \times 10^{6} \mathrm{spores} / \mathrm{ml}$ and incubated at $30^{\circ} \mathrm{C}$ for $72 \mathrm{~h}$. Each cultivation was performed in biological triplicates. Cultures were analyzed for their dry biomass production $72 \mathrm{~h}$ after cultivation (A) and pellet diameter (B). The amount of secreted proteins/g dry biomass (C) was analyzed using a Bradford assay. The amount of secreted GlaA (D) was monitored via Western analysis and normalized firstly against the dry biomass for each corresponding strain/condition. A second normalization was conducted where GlaA was reported relative to the control strain MF31.2 at each respective condition. Students $t$-test was used for significance determination and $p$-values are reported.

\section{Overexpression of arfA Results in Increased Localisation of GlaA at Septa}

When conducting the above assessment of morphology and GlaA localization, an unexpected observation was the detection of GlaA-dtomato at hyphal septa (Figure 6A). Fluorescent signal was observed at septa in all three isolates (Figure 6A and Supplementary Figure S3). We consequently interrogated whether arfA expression levels had any impact on GlaA at these loci. Titration of doxycycline had no effect on the intensity of GlaA distribution in the control strain MF31.2. Additionally, intensity of the GlaA reporter was not impacted by arfA loss-of-function in isolate MF45.5 (Supplementary Figure S3). However, raising the concentration of doxycycline to $10 \mu \mathrm{g} / \mathrm{ml}$ increased the intensity of the GlaA at septa in both doxycycline controlled arfA expression strains (MF45.5 and FH1.1), suggesting that septal localisation of GlaA may be ArfA-dependent (Figures 6B,C and Supplementary Figure S3). Consequently, we measured the signal intensity at septa in isolates MF31.2 and FH1.1, which indicated fluorescent intensity of the GlaA reporter was indeed higher at septal locations following ArfA overexpression (Figure 6B). Quantification of GlaA abundance from fluorescence data indicated that approximately one third more protein was present at septa in the over-expression isolate when compared to the control strain (Table 2). Although these data do not demonstrate secretion of GlaA at hyphal septa, localisation of this protein at these loci is consistent with exocytosis of starch degrading enzymes into septal periplasmic space in the industrial fungus $A$. oryzae (Hayakawa et al., 2011). It is interesting to speculate that increased GlaA secretion in shake flask culture following ArfA 


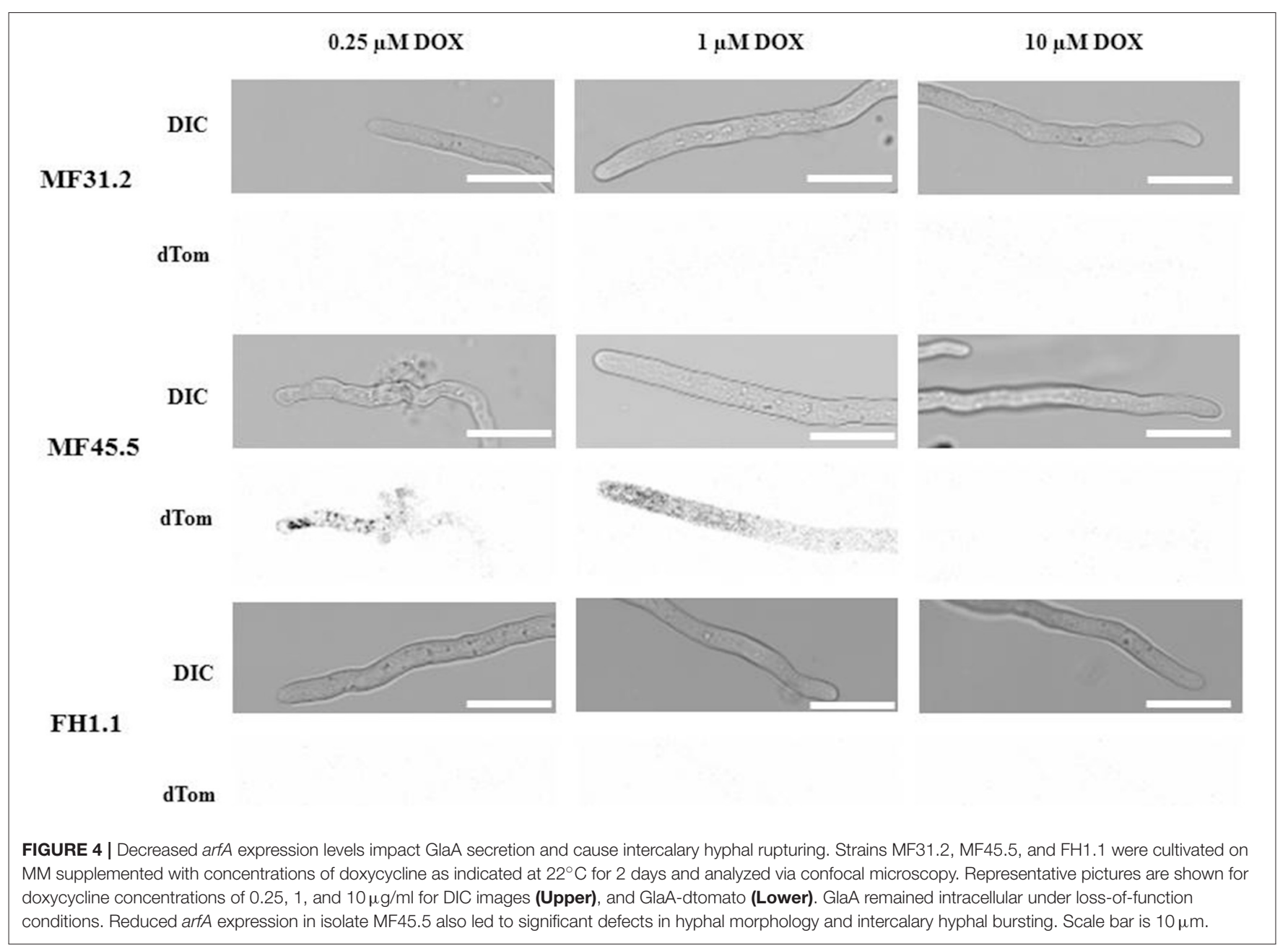

overexpression (Figure 3D) could, at least in part, occur via secretion at hyphal septa.

\section{Depletion and Overexpression of arfA Affects Actin Ring Positioning}

In order to ascertain whether titratable expression of $\operatorname{arf} A$ impacts the cytoskeletal apparatus, we constructed a strain in which expression of arfA was controlled via the Tet-on system and where the actin binding protein AbpA was fluorescently labeled with CFP (MF58.5). The previously published strain MK6.1 (Kwon et al., 2013) expressing AbpA::CFP with no modifications in $\operatorname{arfA}$ gene expression served as respective control (Table 1). The actin ring consists of actin, myosin II, and associated proteins. In the wildtype background (strain MK6.1), this ring is localized about $3 \mu \mathrm{m}$ behind the tip (Figure 7). When both strains were grown on $1 \mu \mathrm{g} / \mathrm{ml}$ doxycycline, the localization of the actin ring was unaltered, although fluorescence was slightly increased in MF58.5 (Figure 7B). The amount of AbpA decreased when ArfA was limited via addition of $0.25 \mu \mathrm{g} / \mathrm{ml}$ doxycycline. Interestingly, the actin ring was shifted approximately $1.2 \mu \mathrm{m}$ toward the tip (Figure 7B). When the concentration was raised to $10 \mu \mathrm{g} / \mathrm{ml}$ doxycycline, higher fluorescence levels compared to the wildtype was observed, and the actin ring shifted again toward the tip $(0.6 \mu \mathrm{m})$ and was more spread toward the apical dome (Figure 7B). Quantitative analyses of fluorescent intensity at either 3 or $2 \mu \mathrm{m}$ confirmed this actin ring shift toward the hyphal tip in $\operatorname{arfA}$ conditional expression mutants (Supplementary Figure S4). From the obtained images we measured and calculated the base area of the apical dome, showing that the area was reduced during downregulation and overexpression of $\operatorname{arf} A$ (Table 3). These data indicate that titration of arfA expression impacts the $A$. niger cytoskeleton in addition to the secretory pathway.

\section{ArfA Does Not Localize With the ER or at the Hyphal Tip in A. niger}

Arf GTPases can localize to a multiple subcellular loci, for example specific regions of the Golgi (Stearns et al., 1991; Lee and Shaw, 2008), and dynamic spots of the plasma membrane (Donaldson, 2003; Smaczynska-de Rooij et al., 2008). In order to establish the subcellular localisation of ArfA, we generated various isolates with fluorescently labeled components of the secretory system. Additionally, the endogenous arfA 


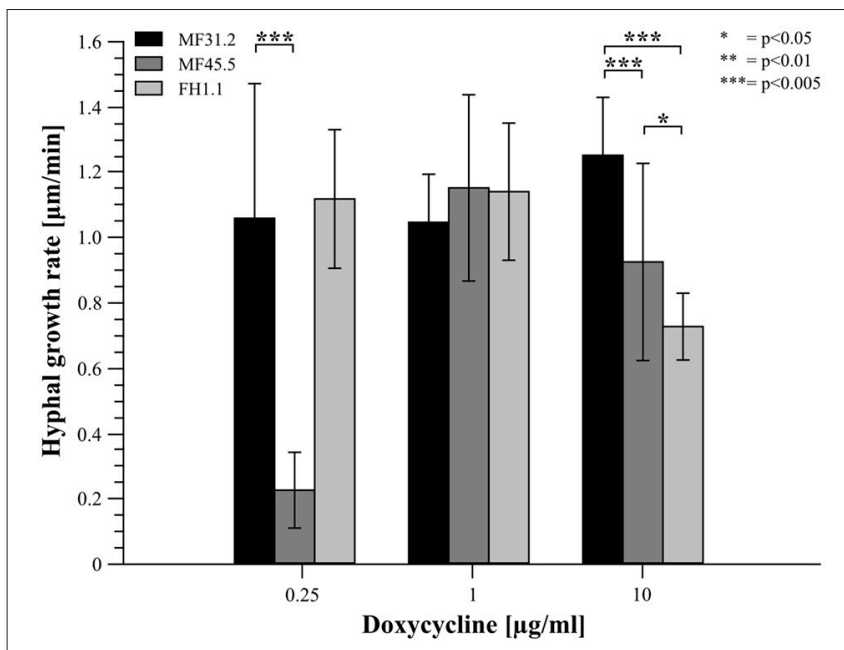

FIGURE 5 | Elevated and reduced arfA expression decreases the growth rate of $A$. niger hyphae. Strains MF31.2, MF45.5, and FH1.1 were cultivated on MM supplemented with different concentrations of doxycycline as indicated, and cultivated at $22^{\circ} \mathrm{C}$ for 2 days. Hyphae were analyzed via confocal time lapse microscopy. Individual hyphae were analyzed in single stacks every $20 \mathrm{~s}$ for $5 \mathrm{~min}$. The hyphal growth rate of at least 10 individual hyphae from two independent experiments was analyzed via ImageJ. Students $t$-test was used for significance determination as indicated.

gene was replaced with a C-terminally dtomato tagged $\operatorname{arfA}$ gene. C-terminal tagging was chosen, because it has been demonstrated in A. nidulans that $\mathrm{N}$-terminal myristoylation is crucial for correct localization of ArfA (Lee and Shaw, 2008). ArfA tagging was conducted in previously published $A$. niger reporter strains, in which distinct steps of the secretory pathway have been fluorescently labeled (Table 1 and Figure 8): (i) the ER (GlaA-GFP-HDEL), (ii) Golgi (GmtA-YFP), and (iii) postGolgi carriers in which the v-SNARE SncA is fluorescently tagged (SncA-GFP) (Carvalho et al., 2011; Kwon et al., 2011). Additionally, a strain carrying only the ArfA-dtomato expression construct (MF56.7) was constructed, to exclude the possibility that combined expression of two fluorescently labeled proteins of the secretory pathway interferes with the localisation of ArfA. All strains were viable and produced spores which were able to grow on selective medium (data not shown), and did not display any defects in standard growth assays using solid media (data not shown), suggesting that replacing the essential arfA with the arfA-dtomato led to functional ArfA-dtomato fusion protein.

Subcellular localisation analyses of ArfA reporter strains showed a punctuate localization of this protein within growing hyphae (Figure 8). ArfA is not distributed to the hyphal tip (Figure 8), an observation supported by linescan analyses along the hyphal length (Supplementary Figure S5). As shown in Figure 8, ArfA does not co-localize with the ER marker. We noted partial co-localization of ArfA with Golgi associated GmtA and with post Golgi carries as determined by SncA localisation (Figure 8). However, in both these latter experiments, ArfA did not exclusively localize with either marker. These data were surprising, given that the ArfA orthologue Arf1 has been demonstrated to predominantly localize at the Golgi in a diverse range of organisms, including mammals (Stearns et al., 1991), S. cerevisiae (Donaldson and Klausner, 1994; Suda et al., 2018), and A. nidulans (Lee and Shaw, 2008). Our data do not conclusively confirm the sub-cellular localization of ArfA in A. niger, but are suggestive of association at an intercalary hyphal region that is also distinct from the ER. With regards to the observed changes in actin ring position in $A$. niger (Figure 7), Arf3 in yeast, and Arf6 in mammals regulate actin organization by activating GAPs at the plasma membrane (Donaldson, 2003; Smaczynska-de Rooij et al., 2008; Hsu and Lee, 2013). Our localization experiments suggest that this is unlikely to be the case for ArfA in A. niger. We therefore hypothesize that modifications to actin ring position (Figure 7) occur as a consequence of defective secretion following titration of arfA expression.

\section{DISCUSSION}

In this study, we have functionally characterized the A. niger ArfA. The ArfA orthologue, Arf1, has been most extensively studied in the budding yeast $S$. cerevisiae, where this protein controls the formation of vesicle coats at distinct steps in intracellular transport. Arf1 in the GDP-bound form is located in the cytosol and is activated by GEFs Gea1/2 or Sec7, which are located at cis-cisternae and trans-cisternae of the Golgi, respectively (Wright et al., 2014). This positional distribution of Arf1-GEFs enables differential effector recruitment by GTPbound Arf1, specifically the COPI vesicles at cis-cisternae, which are required for recycling of Golgi glycoslylation enzymes, and clathrin-coated vesicles at trans-cisternae, which facilitate carrier formation (Suda et al., 2018). Such control of vesicle trafficking is essential in yeast, as double deletion of $A R F 1 / 2$ is lethal (Stearns et al., 1990). Data generated in this study suggests that ArfA may perform a comparable regulatory function in A. niger. Firstly, a complementation approach using $S$. cerevisiae confirmed that this protein is highly likely to function as an ADP-ribosylation factor that can regulate vesicle trafficking in yeast. Next, by putting the arfA gene under control of the Tet-on system in $A$. niger, we were able to concomitantly mimic and analyse downregulation, overexpression and wildtype phenotypes. These loss-of-function analyses demonstrated this gene is also essential in $A$. niger. Subsequent phenotypic screens of $A$. niger arfA conditional expression isolates suggested that the ArfA protein plays a critical regulatory role in secretion, as loss- and gain-of-function isolates were sensitive to various secretory stressors. These data are consistent with defective vesicle trafficking and secretion in ARF1 mutants of different fungi such as $S$. cerevisiae, C. albicans, A. nidulans, and M. circinelloides (Stearns et al., 1991; Lee and Shaw, 2008; Labbaoui et al., 2017; Patiño-Medina et al., 2017). Interestingly, there are deviations in the relative importance of Arfs among Ascomycetes. For example, in contrast to this study, functional analyses of Arf1-3 in C. albicans demonstrated that Arf2, but not Arf1, was essential for cell viability (Labbaoui et al., 
A

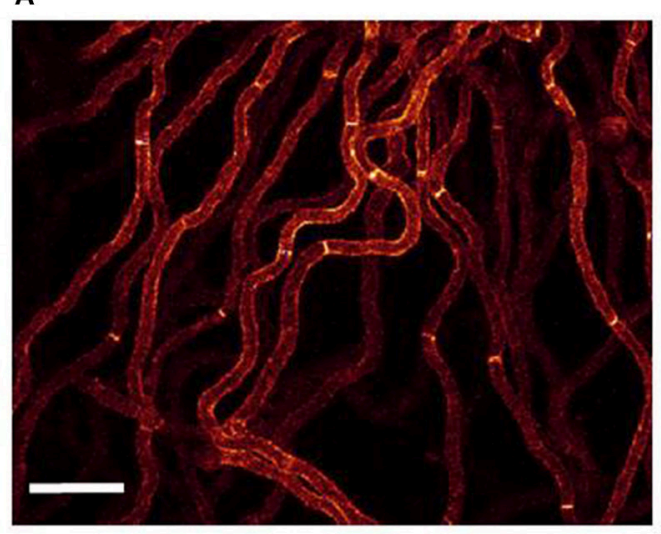

B

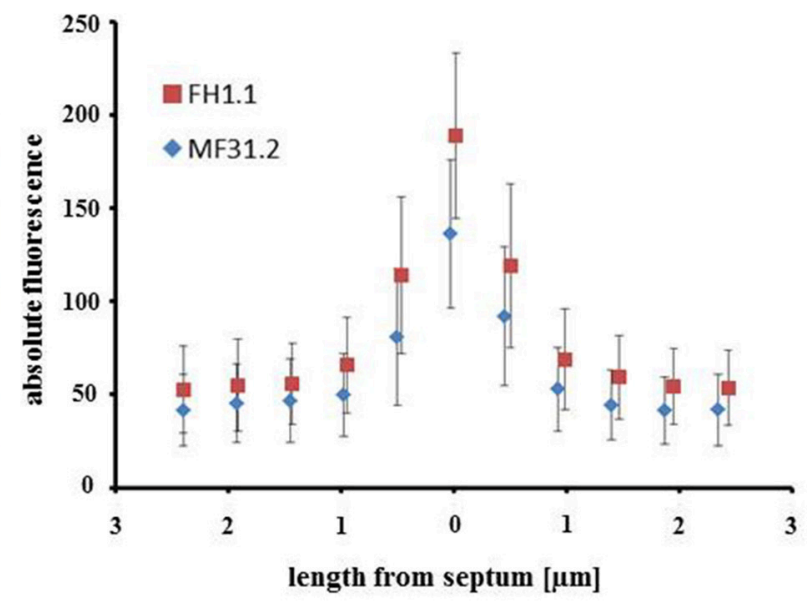

C

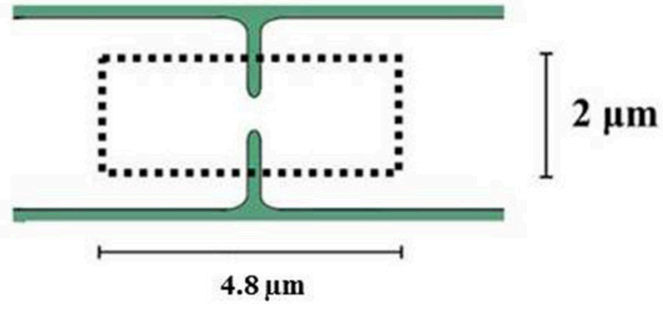

FIGURE 6 | Localisation of GlaA at the hyphal septa increases following arfA overexpression. Strains expressing a GlaA-dtomato reporter protein, and enabling doxycycline mediated wildtype, reduced, and overexpression of arfA (MF31.2, MF45.5, and FH1.1, respectively), were grown at $22^{\circ} \mathrm{C}$ for 2 days on MM plates. After $1 \mathrm{~h}$ of incubation with liquid MM supplemented with respective concentrations of doxycycline, confocal microscopy of GlaA-dtomato was performed and Z-stack series were taken (dark red = low GlaA-dtomato signal, bright yellow = high GlaA intensity signal. (A) Exemplar fluorescent image of growing colonies demonstrated GlaA localizes to hyphal septa in the control and overexpression strain FH1.1 using $10 \mu \mathrm{g} / \mathrm{ml}$ doxycycline. (B) Septum localized GlaA-dtomato fluorescence of 12 hyphal septa from projected Z-stacks was quantified for MF31.2 and FH1.1 grown in the presence of $10 \mu \mathrm{g} / \mathrm{ml}$ doxycycline, which confirmed increased expression of GlaA, especially at the hyphal septum following arfA overexpression. Standard error bars are shown from 12 replicates. A schematic overview of the region analyzed in this analysis is depicted in (C), whereby we took the septum as the central point and measured up to $2.4 \mu \mathrm{m}$ from this point either side, giving a total length of $4.8 \mu \mathrm{m}$.

TABLE 2 | Quantitative abundance of GlaA at hyphal septum determined from fluorescent microscopy.

\begin{tabular}{lccc}
\hline \multirow{2}{*}{ Strain } & \multicolumn{3}{c}{ GlaA Abundance } \\
\cline { 2 - 4 } & Adaxial & Abaxial & Total \\
\hline MF31.2 & 149.2 & 149.5 & 298.7 \\
FH1.1 & 192.6 & 204.8 & 397.4 \\
\hline
\end{tabular}

Septa were taken as central points and absolute fluorescence was measured over a length of $2.4 \mu \mathrm{m}$ in either adaxial or abaxial direction (Figures $\mathbf{6 B}, \mathbf{C}$ ). GlaA concentration was approximated based on polynomial function of the 6th order. Integrals of these functions were determined over the length of $2.4 \mu \mathrm{m}$. These values were used as proxy measures for GlaA abundance, indicating that the arfA over-expression isolate had approximately a third more GlaA at hyphal septa when compared to MF31.2 control.

2017). It is plausible that these discrepancies can be related to subtle differences in Arf function between species, or the disparate lifestyles or environmental niches in which they inhabit.

Interestingly, titratable expression of $\operatorname{arf} A$ resulted in numerous effects during $A$. niger growth in submerged culture, with colony macromorphology, total protein secretion, and extracellular titres of glucoamylase variously impacted. Increased arfA expression led to reduced pellet diameter, a dispersed hyphal morphology, and increased total protein secretion. Elevated titres of secreted protein in an over-expression isolate that concomitantly demonstrates sensitivity to secretory stress was surprising, and may be due to modifications in colony macromorphology under these conditions. For example, in filamentous fungal cell factories, increased protein secretion often correlates with a dispersed morphology as the number of hyphal tips/biomass increases. Numerous techniques, including generation of temperature sensitive mutants (Kwon et al., 2013), and addition of inorganic insoluble micro particles (Wucherpfennig et al., 2012), have been developed to achieve this colony macromorphology in submerged culture. We hypothesize that careful titration of arfA expression may enable optimized morphology in submerged culture, which ultimately may provide novel avenues for improved titres of total secreted protein.

In contrast to over-expression strains, loss-of-function mutants displayed comparable pellet diameter and total 


\section{A}

Doxycycline

$[\mu \mathrm{g} / \mathrm{ml}]$

0.25
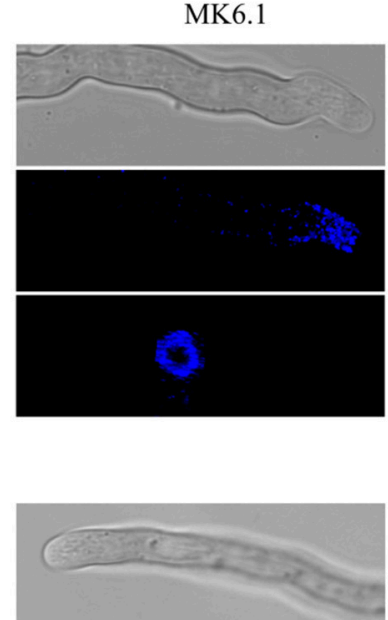

1
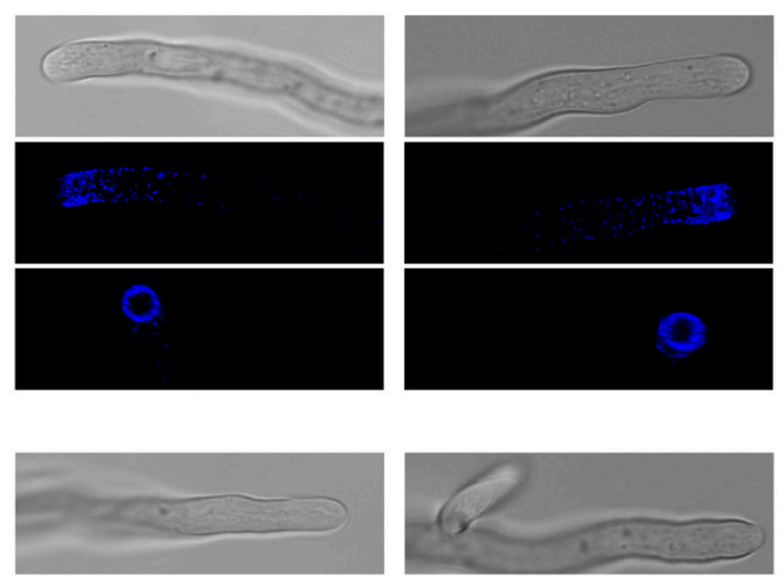

10
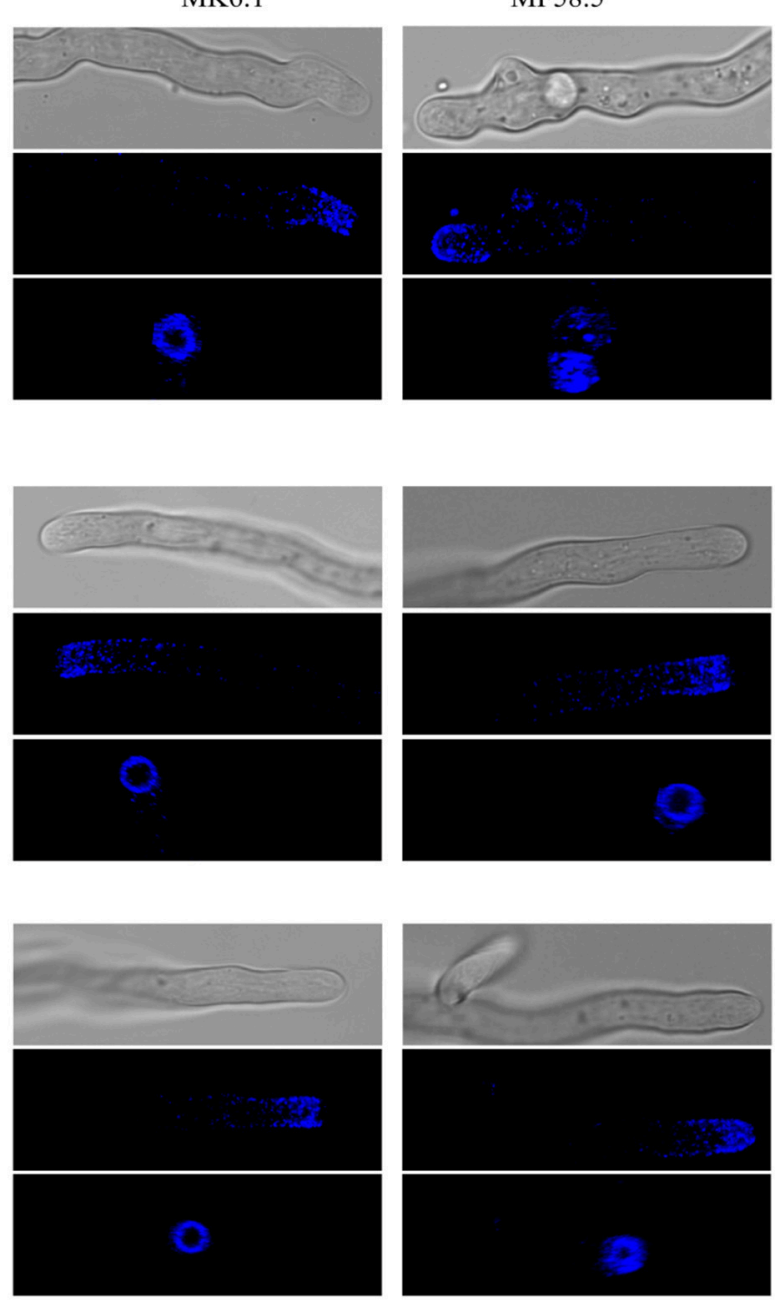

B
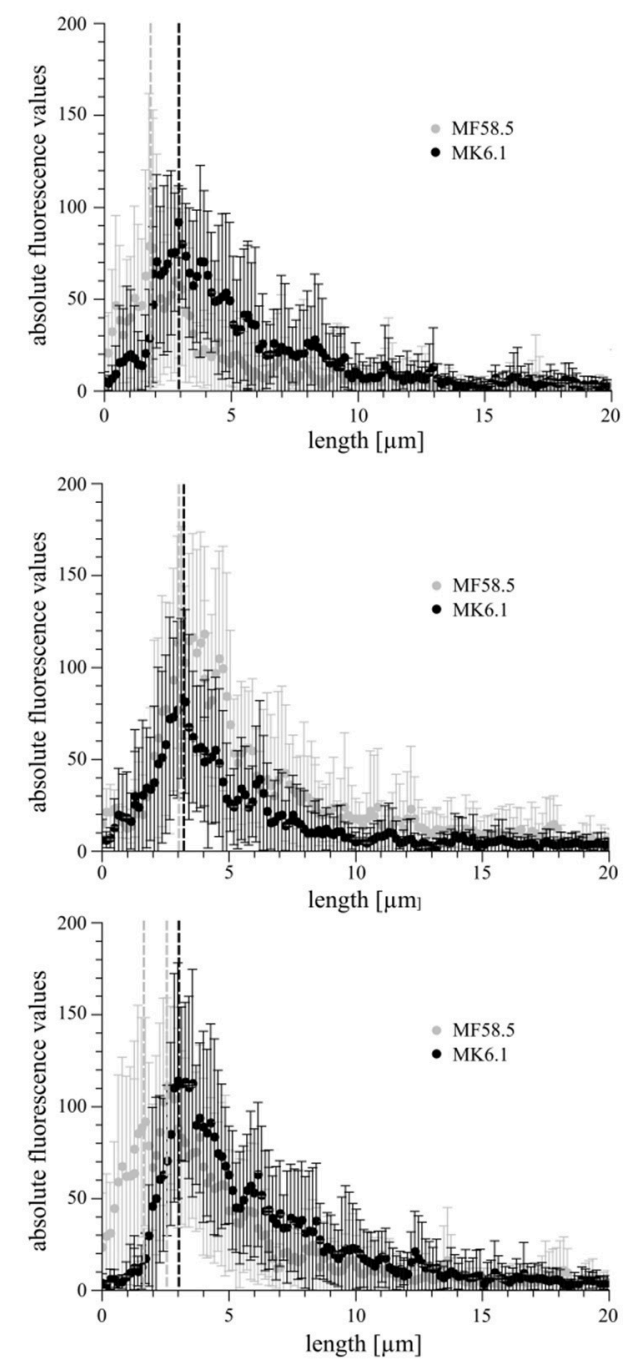

FIGURE 7 | Modifications to arfA expression shift the actin ring toward the hyphal apex. In order to assess the location of the endocytic actin ring, strains MF58.8 and MK6.1 were utilized, which both express the actin binding protein AbpA tagged with cyan fluorescent protein (AbpA::CFP). Isolate MK6.1 was used as control to assess the actin ring position under native arfA expression. Strain MF58.5 has the native arfA deleted, and titratable arfA expression using the Tet-on system. Isolates were cultivated at $22^{\circ} \mathrm{C}$ for 2 days on MM supplemented with various concentrations of doxycycline and analyzed via confocal microscopy. Z-stacks of 15 individual hyphal tips were taken (A), and representative pictures of DIC (upper panels) and projected 3D images of Z-stacks (middle and bottom panels) are shown. These data indicated structural disruption of the actin ring following reduced/increased arfA expression, and a clear shift of AbpA::CFP toward the hyphal apex. Quantification of AbpA::CFP fluorescence along the cell membrane $20 \mu \mathrm{m}$ from the tip apex (B) revealed a shift in actin ring position in ArfA loss- and gain-of-function mutants. Fifteen hyphae/strain/condition were assessed, with bars depicting standard error. Dotted lines denote the predicted position of the actin ring. Note two possible positions are shown for MF58.5 under $10 \mu \mathrm{g} / \mathrm{ml}$ dox due to deterioration of ring structure observed in (A).

secreted protein, yet significantly reduced glucoamylase secretion during submerged culture. Microscopic examination provided two possible explanations for these observations. Firstly, fluorescently labeled GlaA remained intracellular, which is consistent with defective secretion following reduction of ArfA levels. Secondly, reduced glucoamylase secretion may also be attributed to loss of cell viability following intercalary hyphal rupturing.

These data highlight the complex interplay between defects in secretion, aberrant growth rates, morphological changes, and colony macromorphology following titration of arfA expression. Consequently, increased characterization of Arfs, and the phenotypic consequences of their mis-expression in other 
filamentous fungi, will complement the extensive work already conducted in S. cerevisiae and mammals.

Intriguingly, fluorescent microscopy demonstrated that the position of the actin ring at the hyphal tip is modified following titratable arfA expression in $A$. niger (Figure 7). Arfs are known to play important regulatory roles in actin dynamics in a range of organisms. For example, in S. cerevisiae, Arf3 is required for organization of actin cables, cortical patches and endocytosis (Lambert et al., 2007). Arf3 regulates the GTPase activating protein $\mathrm{Bud} 2 \mathrm{p}$ during axial budding (Hsu and Lee, 2013), which is a component of a module with the GTPase Bud1, and the GTP/GDP exchange factor Bud5. This module activates Cdc24, which subsequently activates Cdc42, which in turn regulates actin dynamics necessary for polar growth (Hsu and Lee, 2013). The Arf3 orthologue in mammals, Arf6, also plays a regulatory role in actin polymerization (D'SouzaSchorey et al., 1997). Data presented in this study demonstrates that under native arfA expression levels, the actin ring is positioned about $3 \mu \mathrm{m}$ behind the hyphal apex, but moves toward the tip when $\operatorname{arfA}$ is downregulated (about $1.2 \mu \mathrm{m}$ ) or upregulated (about $0.6 \mu \mathrm{m}$ ). It is possible that the shift in actin ring position following titration of arfA expression occurs due to defective delivery of necessary molecular markers to the tip. Indeed, in yeast, the position of actin patches is dependent on lipid domains (Cortesio et al., 2015), and the composition of these signaling lipids might be impacted by modifications in secretory pathway following arfA misexpression. Alternatively, as is the case for Arf3 in S. cerevisiae and Arf6 in mammals, there might be a direct interaction of ArfA with regulatory modules that are necessary for actin organization in A. niger. However, given that Arf3 and Arf6 localize to the plasma membrane (Donaldson, 2003; Smaczynska-de Rooij et al., 2008), and ArfA in this study localized to intercalary region of growing hyphae (Figure 8), we hypothesize that modifications in actin position are due to defective trafficking of lipid domains or membrane and cell wall components (Figure 9).

Nevertheless, our study does suggest that changes in actin ring position could partially account for the phenotypic consequences of $\operatorname{arfA}$ mis-expression. The actin ring is the site of endocytosis, and the location of this cytoskeletal apparatus at the hyphal tip is vital for polar growth (Taheri-Talesh et al., 2008). In A. nidulans, the actin ring is maintained precisely $1-2 \mu \mathrm{m}$ behind the hyphal apex, even in rapidly growing hyphae (TaheriTalesh et al., 2008). This spatial maintenance is likely mediated by actin cables, and occurs despite the short lifespan of actin patches from which the ring is composed $[<1$ min (TaheriTalesh et al., 2008)]. Such spatial control of the actin ring enables recycling of exocytic vesicle membrane and v-SNAREs and cell wall synthesizing enzymes as they move to posterior regions of the hyphae due to plasma membrane growth. This removal from posterior regions of the hyphae ensures markers for vesicle fusion are located exclusively at the apex, and stops plasma membrane expansion due to secretory vesicle membranes (Taheri-Talesh et al., 2008; Takeshita et al., 2013). Further importance of the tightly maintained subcellular location of the actin ring in fungal hyphae has been predicted by
TABLE 3 | arfA expression influences the base area of the apical dome.

\begin{tabular}{lccc}
\hline Strain & \multicolumn{3}{c}{ Doxycycline $[\mu \mathrm{g} / \mathrm{ml}]$} \\
\cline { 2 - 4 } & $\mathbf{0 . 2 5}$ & $\mathbf{1}$ & $\mathbf{1 0}$ \\
\hline MK6.1 & $\mathbf{3 5 . 6 9} \pm \mathbf{2 . 6 1}$ & $\mathbf{3 5 . 8 8} \pm \mathbf{4 . 3 6}$ & $\mathbf{3 5 . 9 0} \pm \mathbf{4 . 9 8}$ \\
& $100 \pm 7.32$ & $100 \pm 12.17$ & $100 \pm 13.87$ \\
MF58.5 & $\mathbf{2 3 . 9 6} \pm \mathbf{4 . 3 0}$ & $\mathbf{3 7 . 1 9} \pm \mathbf{6 . 7 3}$ & $\mathbf{2 7 . 7 6} \pm \mathbf{2 . 9 3}$ \\
& $67.15 \pm 12.05$ & $103 \pm 18.76$ & $77.31 \pm 8.18$ \\
\hline
\end{tabular}

Calculation was done using values from eight individual hyphae of MF58.5 and MK6.1, which were used for the analysis of actin ring position in Figure 6. Area is given in [ $\mu \mathrm{m}^{2}$ ] (bold) and respective percentage corresponding to MK6.1 (italics).

mathematical growth models using C. albicans, which have subsequently been verified using quantitative measurements of fluorescently labeled components of the exocytic and endocytic machinery (Caballero-Lima et al., 2013). Consequently, the spatial control of the actin ring behind the hyphal apex (1$2 \mu \mathrm{m}$ ) in A. nidulans (Taheri-Talesh et al., 2008), and $3 \mu \mathrm{m}$ in A. niger (Figure 7) may ensure that endocytosis and recycling of cell wall synthesizing enzymes occurs at a precise location that maintains polarized growth while avoiding hyphal swelling. We hypothesize that the observed defects in growth rates and morphology in arfA loss- and gain-of-function mutants can be firstly attributed to defects in secretion, which may either cause, and/or be compounded by, modulation of actin ring position (Figure 9).

This study also suggests that the $A$. niger glucoamylase might not only be secreted at the hyphal tip, but also at hyphal septa as previously suspected (Gordon et al., 2000). This is consistent with studies using $A$. oryzae, where the major extracellular protein alpha-amylase was observed to localize in the space between the plasma membrane and cell wall at septa [i.e., the septal periplasm (Hayakawa et al., 2011)]. Septal exocytosis is required for secondary cell wall thickening, intercalary hyphal growth, and branch initiation in filamentous fungi (Hayakawa et al., 2011; Read, 2011). Interestingly, microscopic monitoring of fluorescent dye uptake has demonstrated that exocytosis is also coupled with endocytosis at intercalary loci in a variety of fungi, including A. nidulans (Fischer-Parton et al., 2000). In our study, overexpression of arfA resulted in increased septal GlaA localisation. It is thus possible that ArfA is also required for normal exocytic and/or endocytic processes at the hyphal septum.

In conclusion, we have functionally characterized the essential protein ArfA in the filamentous cell factory A. niger. ArfA has critical implications for fungal growth rates, and hyphal/colony morphology. Moreover, by titrating arfA expression at different levels, we were able to increase the amount of secreted proteins. Actin ring position was also impacted by arfA expression levels, which might impact plasma membrane and cell wall growth at the hyphal apex. Finally, our data suggest that septal secretion may occur in $A$. niger. Further studies are required to determine if secretion at septa does indeed occur in 


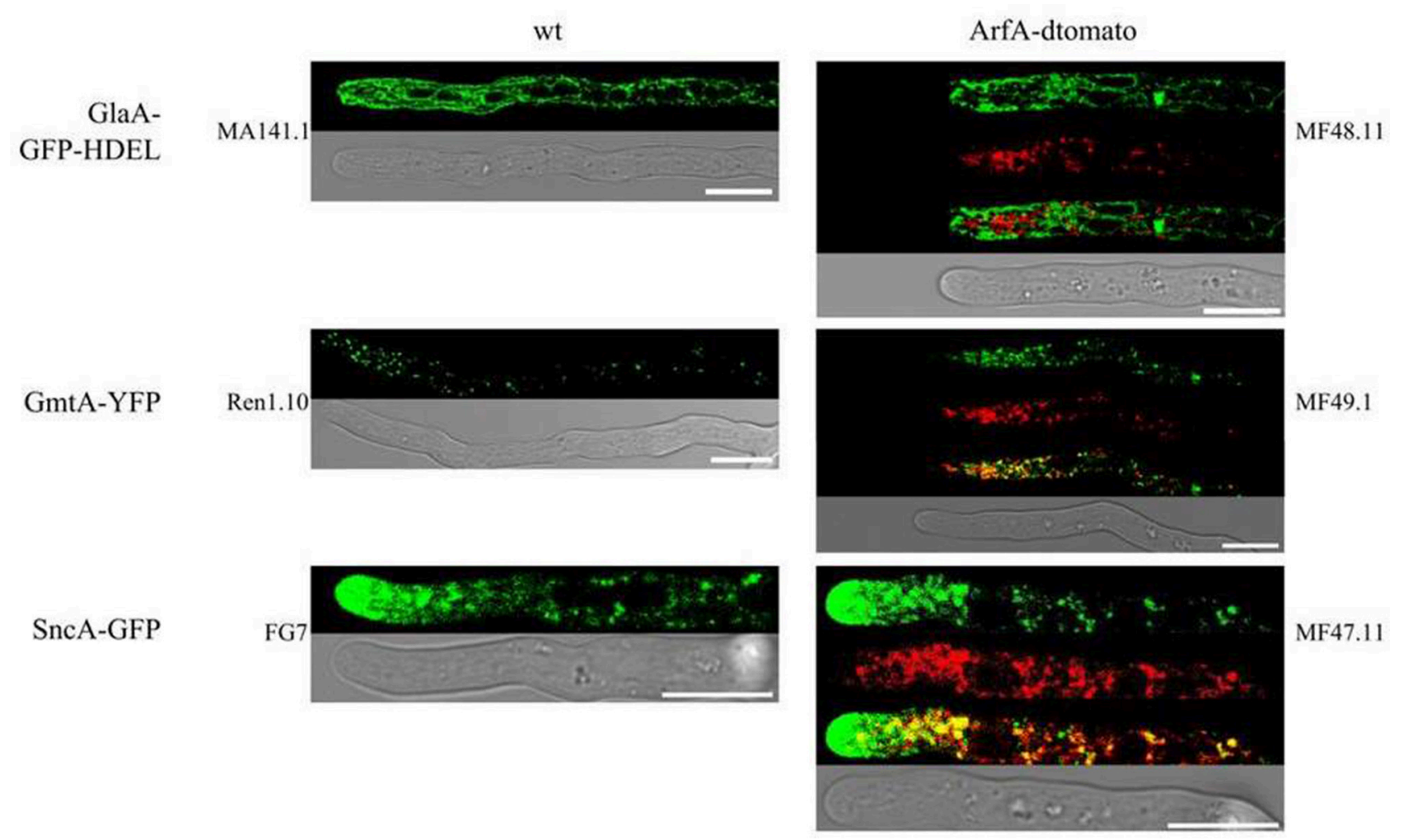

FIGURE 8 | ArfA does not localize with the ER or at the hyphal tip in A. niger. Strains were cultivated on MM agar plates at $22^{\circ} \mathrm{C}$ for 2 days. Co-localization studies utilized strains which each have a specific component of the secretory system tagged, including the ER (GlaA-GFP-HDEL, isolate MA141.1), Golgi (GmtA-YFP, isolate Ren1.10) and post-Golgi carriers (SncA-GFP, isolate FG7). Localization of each reporter demonstrated expected localization of each secretory component (left panels). Co-localization experiments were conducted by expressing an ArfA-dtomato reporter in isolates MF48.11, MF49.1, and MF47.11, respectively, which revealed ArfA does not localize to the ER or the hyphal tip. Partial co-localization was observed between ArfA and Golgi and Golgi carriers. Scale bars on all images represent $10 \mu \mathrm{m}$.
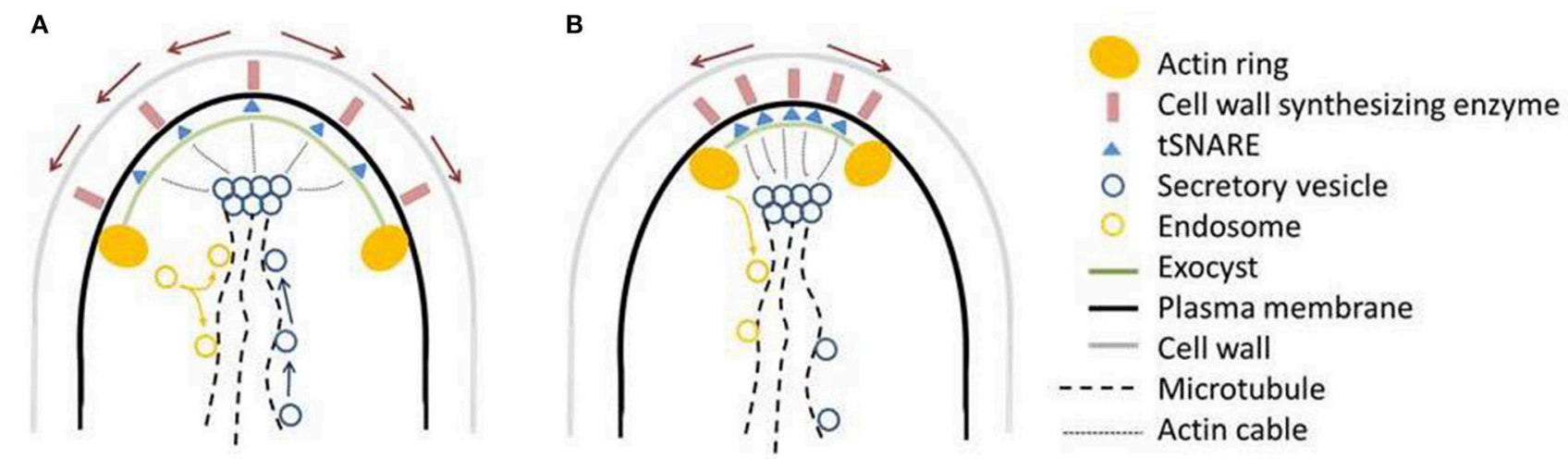

FIGURE 9 | Working model for ArfA dependent secretion, actin ring positioning, hyphal growth, and morphology. (A) In wildtype hyphal tips, secretory vesicles containing cell end markers, wall synthesizing enzymes and other cargo move along microtubules and actin cables to the Spitzenkörper, and subsequently are fused to the plasma membrane by the exocyst/SNARE proteins, releasing hydrolytic/cell wall synthesizing enzymes. The actin ring is fixed a constant position of about $3 \mu \mathrm{m}$ from the apex. Growth of the plasma membrane moves SNAREs/cell wall synthesizing enzymes to the posterior of the hypha (red arrows), which become endocytosed at the actin ring. Endosomes either recycle the contents back to the plasma membrane, presumably via the Spitzenkörper, or back to the anterior of the cell along microtubules as shown for A. nidulans (Taheri-Talesh et al., 2008). (B) Following a reduction or increase in ArfA levels, the secretory pathway is defective, disrupting supply of markers, enzymes, and other cargo. Either due to direct ArfA regulation, or due to this disruption of secretory cargo, the actin ring shifts an average of 0.6-1.2 $\mu \mathrm{m}$ toward the hyphal tip. Both defective vesicle formation, and actin ring position, may synergistically contribute to the pleiotropic phenotypic consequences of arfA mis-expression. For further discussion see main text. 
A. niger, and whether this process could be harnessed in industrial applications for improved titres of useful proteins.

\section{AVAILABILITY OF DATA AND MATERIALS}

Data and materials generated during and/or analyzed in the current study are available from the corresponding author on request.

\section{AUTHOR CONTRIBUTIONS}

VM and MF conceived of the study; MF, OK, and CK conducted experiments; VM, MF, and TC analyzed and interpreted data, generated figures, and wrote the

\section{REFERENCES}

Arentshorst, M., Niu, J., and Ram, A. F. J. (2015). "Efficient generation of Aspergillus niger knock out strains by combining NHEJ mutants and a split marker approach," in Genetic Transformation Systems in Fungi, Vol. 1, eds M. van den Berg and K. Maruthachalam (Cham: Springer International Publishing), 263-272.

Arentshorst, M., Ram, A. F. J., and Meyer, V. (2012). Using non-homologous end-joining-deficient strains for functional gene analyses in filamentous fungi. Methods Mol. Biol. 835, 133-150. doi: 10.1007/978-1-61779501-5_9

Baker Brachmann, C., Davies, A., Cost, G. J., Caputo, E., Li, J., Hieter, P., et al. (1998). Designer deletion strains derived from Saccharomyces cerevisiae S288C: a useful set of strains and plasmids for PCR-mediated gene disruption and other applications. Yeast 14, 115-132. doi: 10.1002/(SICI)10970061(19980130)14:2<115::AID-YEA204>3.0.CO;2-2

Boeke, J. D., LaCroute, F., and Fink, G. R. (1984). A positive selection for mutants lacking orotidine-5'-phosphate decarboxylase activity in yeast: 5 -fluoro-orotic acid resistance. Mol. Gen. Genet. 197, 345-346.

Bos, C. J., Debets, A. J., Swart, K., Huybers, A., Kobus, G., and Slakhorst, S. M. (1988). Genetic analysis and the construction of master strains for assignment of genes to six linkage groups in Aspergillus niger. Curr. Genet. 14, 437-443. doi: $10.1007 / \mathrm{BF} 00521266$

Caballero-Lima, D., Kaneva, I. N., Watton, S. P., Sudbery, P. E., and Craven, C. J. (2013). The spatial distribution of the exocyst and actin cortical patches is sufficient to organize hyphal tip growth. Eukaryotic Cell 12, 998-1008. doi: 10.1128/EC.00085-13

Cairns, T. C., Studholme, D. J., Talbot, N. J., and Haynes, K. (2016). New and improved techniques for the study of pathogenic fungi. Trends Microbiol. 24, 35-50. doi: 10.1016/j.tim.2015.09.008

Carvalho, N. D., Arentshorst, M., Weenink, X. O., Punt, P. J., van den Hondel, C., and Ram, A. F. (2011). Functional YFP-tagging of the essential GDP-mannose transporter reveals an important role for the secretion related small GTPase SrgC protein in maintenance of Golgi bodies in Aspergillus niger. Fungal Biol. 115, 253-264. doi: 10.1016/j.funbio.2010. 12.010

Cortesio, C. L., Lewellyn, E. B., and Drubin, D. G. (2015). Control of lipid organization and actin assembly during clathrin-mediated endocytosis by the cytoplasmic tail of the rhomboid protein Rbd2. Mol. Biol. Cell 26, 1509-1522. doi: 10.1091/mbc.E14-11-1540

D'Souza-Schorey, C., Boshans, R. L., McDonough, M., Stahl, P. D., and Van Aelst, L. (1997). A role for POR1, a Rac1-interacting protein, in ARF6-mediated cytoskeletal rearrangements. $Е M B O J .16,5445-5454$.

Donaldson, J. G. (2003). Multiple roles for Arf6: sorting, structuring, and signaling at the plasma membrane. J. Biol. Chem. 278, 41573-41576. doi: $10.1074 / j b c . R 300026200$ manuscript; Lars Barthel is acknowledged for helping with the statistical analyses. All authors read and approved the final manuscript.

\section{ACKNOWLEDGMENTS}

We acknowledge support by the German Research Foundation and the Open Access Publication Funds of Technische Universität Berlin.

\section{SUPPLEMENTARY MATERIAL}

The Supplementary Material for this article can be found online at: https://www.frontiersin.org/articles/10.3389/fmicb. 2018.00878/full\#supplementary-material

Donaldson, J. G., and Klausner, R. D. (1994). ARF: a key regulatory switch in membrane traffic and organelle structure. Curr. Opin. Cell Biol. 6, 527-532. doi: 10.1016/0955-0674(94)90072-8

Echauri-Espinosa, R. O., Callejas-Negrete, O. A., Roberson, R. W. Bartnicki-García, S., and Mouriño-Pérez, R. R. (2012). Coronin is a component of the endocytic collar of hyphae of Neurospora crassa and is necessary for normal growth and morphogenesis. PLOS ONE 7:e38237. doi: 10.1371/journal.pone.0038237

Fiedler, M. R., Lorenz, A., Nitsche, B. M., van den Hondel, C. A., Ram, A. F., and Meyer, V. (2014). The capacity of Aspergillus niger to sense and respond to cell wall stress requires at least three transcription factors: RlmA, MsnA and CrzA. Fungal Biol. Biotechnol. 1:5. doi: 10.1186/s40694-014-0005-8

Fiedler, M. R. M. (2017). System- und zellbiologische Untersuchungen zum polaren Wachstum in Aspergillus niger, $\mathrm{PhD}$, Technische Universität Berlin, Berlin.

Fiedler, M. R. M., Gensheimer, T., Kubisch, C., and Meyer, V. (2017). HisB as novel selection marker for gene targeting approaches in Aspergillus niger. BMC Microbiol. 17:57. doi: 10.1186/s12866-017-0960-3

Fiedler, M. R. M., Nitsche, B. M., Franziska, W., and Meyer, V. (2013). "Aspergillus: a cell factory with unlimited prospects," in Applications of Microbial Engineering, eds V. K. Gupta, M. Schmoll, and M. Maki (Boca Raton, FL : CRC Press, Taylor \& Francis Group), 1-51.

Fischer-Parton, S., Parton, R. M., Hickey, P. C., Dijksterhuis, J., Atkinson, H. A., and Read, N. D. (2000). Confocal microscopy of FM4-64 as a tool for analysing endocytosis and vesicle trafficking in living fungal hyphae. J. Microsc. 198, 246-259. doi: 10.1046/j.1365-2818.2000.00708.x

Franco, M., Chardin, P., Chabre, M., and Paris, S. (1996). Myristoylation-facilitated binding of the G protein ARF1GDP to membrane phospholipids is required for its activation by a soluble nucleotide exchange factor. J. Biol. Chem. 271, 1573-1578. doi: 10.1074/jbc.271.3.1573

Gibson, D. G. (2011). Enzymatic assembly of overlapping DNA fragments. Meth. Enzymol. 498, 349-361. doi: 10.1016/B978-0-12-385120-8.00015-2

Gillingham, A. K., and Munro, S. (2007). The small G proteins of the Arf family and their regulators. Annu. Rev. Cell Dev. Biol. 23, 579-611. doi: 10.1146/annurev.cellbio.23.090506.123209

Gordon, C. L., Khalaj, V., Ram, A. F. J., Archer, D. B., Brookman, J. L., Trinci, A. P. J., et al. (2000). Glucoamylase::green fluorescent protein fusions to monitor protein secretion in Aspergillus niger. Microbiology 146, 415-426. doi: 10.1099/00221287-146-2-415

Green, M. R., and Sambrook, J. (2012). Molecular Cloning : A Laboratory Manual. Cold Spring Harbor, NY : Cold Spring Harbor Laboratory Press.

Grimm, L. H., Kelly, S., Krull, R., and Hempel, D. C. (2005). Morphology and productivity of filamentous fungi. Appl. Microbiol. Biotechnol. 69, 375-384. doi: 10.1007/s00253-005-0213-5

Hayakawa, Y., Ishikawa, E., Shoji, J., Nakano, H., and Kitamoto, K. (2011). Septumdirected secretion in the filamentous fungus Aspergillus oryzae. Mol. Microbiol. 81, 40-55. doi: 10.1111/j.1365-2958.2011.07700.x 
He, X., Li, S., and Kaminskyj, S. G. W. (2014). Characterization of Aspergillus nidulans $\alpha$-glucan synthesis: roles for two synthases and two amylases. Mol. Microbiol. 91, 579-595. doi: 10.1111/mmi.12480

Helmschrott, C., Sasse, A., Samantaray, S., Krappmann, S., and Wagener, J. (2013). Upgrading fungal gene expression on demand: improved systems for doxycycline-dependent silencing in Aspergillus fumigatus. Appl. Environ. Microbiol. 79, 1751-1754. doi: 10.1128/AEM.03626-12

Hsu, J.-W., and Lee, F.-J. S. (2013). Arf3p GTPase is a key regulator of Bud2p activation for invasive growth in Saccharomyces cerevisiae. Mol. Biol. Cell 24, 2328-2339. doi: 10.1091/mbc.E13-03-0136

Ishitsuka, Y., Savage, N., Li, Y., Bergs, A., Grün, N., Kohler, D., et al. (2015). Superresolution microscopy reveals a dynamic picture of cell polarity maintenance during directional growth. Sci. Adv. 1:e1500947. doi: 10.1126/sciadv.1500947

Jørgensen, T. R., Goosen, T., Hondel, C. A., Ram, A. F., and Iversen, J. J. (2009). Transcriptomic comparison of Aspergillus niger growing on two different sugars reveals coordinated regulation of the secretory pathway. BMC Genomics 10:44. doi: 10.1186/1471-2164-10-44

Kwon, M. J., Arentshorst, M., Fiedler, M., de Groen, F. L. M., Punt, P. J., Meyer, V., et al. (2014). Molecular genetic analysis of vesicular transport in Aspergillus niger reveals partial conservation of the molecular mechanism of exocytosis in fungi. Microbiology 160, 316-329. doi: 10.1099/mic.0.074252-0

Kwon, M. J., Arentshorst, M., Roos, E. D., Van Den Hondel, C. A., Meyer, V., and Ram, A. F. (2011). Functional characterization of Rho GTPases in Aspergillus niger uncovers conserved and diverged roles of Rho proteins within filamentous fungi. Mol. Microbiol. 79, 1151-1167. doi: 10.1111/j.1365-2958.2010.07524.x

Kwon, M. J., Nitsche, B. M., Arentshorst, M., Jorgensen, T. R., Ram, A. F., and Meyer, V. (2013). The transcriptomic signature of RacA activation and inactivation provides new insights into the morphogenetic network of Aspergillus niger. PLoS ONE 8:e68946. doi: 10.1371/journal.pone.0068946

Labbaoui, H., Bogliolo, S., Ghugtyal, V., Solis, N. V., Filler, S. G., Arkowitz, R. A., et al. (2017). Role of Arf GTPases in fungal morphogenesis and virulence. PLoS Pathog. 13:e1006205. doi: 10.1371/journal.ppat.1006205

Lambert, A. A., Perron, M. P., Lavoie, E., and Pallotta, D. (2007). The Saccharomyces cerevisiae Arf3 protein is involved in actin cable and cortical patch formation. FEMS Yeast Res. 7, 782-795. doi: 10.1111/j.1567-1364.2007.00239.x

Lee, S. C., and Shaw, B. D. (2008). Localization and function of ADP ribosylation factor A in Aspergillus nidulans. FEMS Microbiol. Lett. 283, 216-222. doi: 10.1111/j.1574-6968.2008.01174.x

Lew, R. R. (2011). How does a hypha grow? The biophysics of pressurized growth in fungi. Nat. Rev. Microbiol. 9, 509-518. doi: 10.1038/nrmicro2591

Liu, W. (ed.). (2012). "Control of calcium in yeast cells," in Introduction to Modeling Biological Cellular Control Systems, MS\&A (Milano: Springer Milan), 95-122.

Liu, Y., Kahn, R. A., and Prestegard, J. H. (2009). Structure and membrane interaction of myristoylated ARF1. Structure 17, 79-87. doi: 10.1016/j.str.2008.10.020

Lombraña, M., Moralejo, F. J., Pinto, R., and Martín, J. F. (2004). Modulation of Aspergillus awamori thaumatin secretion by modification of bipA gene expression. Appl. Environ. Microbiol. 70, 5145-5152. doi: 10.1128/AEM.70.9.5145-5152.2004

Lo Presti, L., Lanver, D., Schweizer, G., Tanaka, S., Liang, L., Tollot, M., et al. (2015). Fungal effectors and plant susceptibility. Annu. Rev. Plant Biol. 66, 513-545. doi: 10.1146/annurev-arplant-043014-114623

Luini, A., Ragnini-Wilson, A., Polishchuck, R. S., and De Matteis, M. A. (2005). Large pleiomorphic traffic intermediates in the secretory pathway. Curr. Opin. Cell Biol. 17, 353-361. doi: 10.1016/j.ceb.2005.06.012

Meyer, V., Andersen, M. R., Brakhage, A. A., Braus, G. H., Caddick, M. X., Cairns, C. T., et al. (2016). Current challenges of research on filamentous fungi in relation to human welfare and a sustainable bio-economy: a white paper. Fungal Biol. Biotechnol. 3:6. doi: 10.1186/s40694-016-0024-8

Meyer, V., Arentshorst, M., El-Ghezal, A., Drews, A. C., Kooistra, R., van den Hondel C. A. et al. (2007). Highly efficient gene targeting in the Aspergillus niger kusA mutant. J Biotechnol. 128, 770-775. doi: 10.1016/j.jbiotec.2006.12.021

Meyer, V., Fiedler, M., Nitsche, B., and King, R. (2015). The cell factory Aspergillus enters the big data era: opportunities and challenges for optimising product formation. Adv. Biochem. Eng. Biotechnol. 149, 91-132. doi: 10.1007/10_2014_297

Meyer, V., Ram, A. F. J., and Punt, P. J. (2010). "Genetics, genetic manipulation, and approaches to strain improvement of filamentous fungi," in Manual of Industrial Microbiology and Biotechnology, eds R. Baltz, A. Demain, J. Davies, A. Bull, B. Junker, L. Katz, L. Lynd, P. Masurekar, C. Reeves, and H. Zhao (New York, NY: Wiley), 318-329.

Meyer, V., Wanka, F., van Gent, J., Arentshorst, M., van den Hondel, C. A., and Ram, A. F. (2011). Fungal gene expression on demand: an inducible, tunable, and metabolism-independent expression system for Aspergillus niger. Appl. Environ. Microbiol. 77, 2975-2983. doi: 10.1128/AEM.02740-10

Moralejo, F. J., Watson, A. J., Jeenes, D. J., Archer, D. B., and Martín, J. F. (2001). A defined level of protein disulfide isomerase expression is required for optimal secretion of thaumatin by Aspegillus awamori. Mol. Genet. Genomics 266, 246-253. doi: 10.1007/s004380100550

Patiño-Medina, J. A., Maldonado-Herrera, G., Pérez-Arques, C., AlejandreCastañeda, V., Reyes-Mares, N. Y., Valle-Maldonado, M. I., et al. (2017). Control of morphology and virulence by ADP-ribosylation factors (Arf) in Mucor circinelloides. Curr. Genet. doi: 10.1007/s00294-017-0798-0. [Epub ahead of print].

Poon, P. P., Cassel, D., Spang, A., Rotman, M., Pick, E., Singer, R. A., et al. (1999). Retrograde transport from the yeast Golgi is mediated by two ARF GAP proteins with overlapping function. EMBO J. 18, 555-564. doi: $10.1093 / \mathrm{emboj} / 18.3 .555$

Poon, P. P., Wang, X., Rotman, M., Huber, I., Cukierman, E., Cassel, D., et al. (1996). Saccharomyces cerevisiae Gcs1 is an ADP-ribosylation factor GTPase-activating protein. Proc. Natl. Acad. Sci. U.S.A. 93, 10074-10077. doi: 10.1073/pnas.93.19.10074

Punt, P. J., Oliver, R. P., Dingemanse, M. A., Pouweisa, P. H., and van den Hondel, C. A. (1987). Transformation of Aspergillus based on the hygromycin B resistance marker from Escherichia coli. Gene 56, 117-124. doi: 10.1016/0378-1119(87)90164-8

Punt, P. J., van Gemeren, I. A., Drint-Kuijvenhoven, J., Hessing, J. G., van Muijlwijk-Harteveld, G. M., Beijersbergen, A., et al. (1998). Analysis of the role of the gene bipA, encoding the major endoplasmic reticulum chaperone protein in the secretion of homologous and heterologous proteins in black Aspergilli. Appl. Microbiol. Biotechnol. 50, 447-454. doi: 10.1007/s0025300 51319

Ram, A. F. J., and Klis, F. M. (2006). Identification of fungal cell wall mutants using susceptibility assays based on Calcofluor white and Congo red. Nat. Protoc. 1, 2253-2256. doi: 10.1038/nprot.2006.397

Read, N. D. (2011). Exocytosis and growth do not occur only at hyphal tips. Mol. Microbiol. 81, 4-7. doi: 10.1111/j.1365-2958.2011.07702.x

Riquelme, M., Bredeweg, E. L., Callejas-Negrete, O., Roberson, R. W., Ludwig, S. Beltrán-Aguilar, A., et al. (2014). The Neurospora crassa exocyst complex tethers Spitzenkörper vesicles to the apical plasma membrane during polarized growth. Mol. Biol. Cell 25, 1312-1326. doi: 10.1091/mbc.E13-06-0299

Roncero, C., and Durán, A. (1985). Effect of Calcofluor white and Congo red on fungal cell wall morphogenesis: in vivo activation of chitin polymerization. J. Bacteriol. 163, 1180-1185.

Roth, M. G. (1999). Snapshots of ARF1: implications for mechanisms of activation and inactivation. Cell 97, 149-152.

Sambrook, J. F. (1990). The involvement of calcium in transport of secretory proteins from the endoplasmic reticulum. Cell 61, 197-199. doi: 10.1016/0092-8674(90)90798-J

Smaczynska-de Rooij, I. I., Costa, R., and Ayscough, K. R. (2008). Yeast Arf3p modulates plasma membrane PtdIns(4,5)P2 levels to facilitate endocytosis. Traffic 9, 559-573. doi: 10.1111/j.1600-0854.2008.00708.x

Stearns, T., Kahn, R. A., Botstein, D., and Hoyt, M. A. (1990). ADP ribosylation factor is an essential protein in Saccharomyces cerevisiae and is encoded by two genes. Mol. Cell. Biol. 10, 6690-6699. doi: 10.1128/MCB.10.12.6690

Stearns, T., Willingham, M. C., Botstein, D., Kahn, R., a, Tsuchiya, M., Price, S. R., et al. (1991). ADP-ribosylation factor is functionally and physically associated with the Golgi complex. Meth. Enzymol. 267, 1175-1178.

Suda, Y., Kurokawa, K., and Nakano, A. (2018). Regulation of ER-Golgi transport dynamics by GTPases in budding yeast. Front. Cell Dev. Biol. 5:122. doi: $10.3389 /$ fcell.2017.00122 
Taheri-Talesh, N., Horio, T., Araujo-Bazán, L., Dou, X., Espeso, E. A., Pen, M. A., et al. (2008). The tip growth apparatus of Aspergillus nidulans. Mol. Biol. Cell 19, 1439-1449. doi: 10.1091/mbc.E07-05-0464

Takeshita, N., and Fischer, R. (2011). On the role of microtubules, cell end markers, and septal microtubule organizing centres on site selection for polar growth in Aspergillus nidulans. Fungal Biol. 115, 506-517. doi: 10.1016/j.funbio.2011.02.009

Takeshita, N., Mania, D., Herrero, S., Ishitsuka, Y., Nienhaus, G. U., Podolski, M., et al. (2013). The cell-end marker TeaA and the microtubule polymerase AlpA contribute to microtubule guidance at the hyphal tip cortex of Aspergillus nidulans to provide polarity maintenance. J. Cell Sci. 126, 5400-5411. doi: $10.1242 /$ jcs.129841

Takeuchi, M., Ueda, T., Yahara, N., and Nakano, A. (2002). Arf1 GTPase plays roles in the protein traffic between the endoplasmic reticulum and the Golgi apparatus in tobacco and Arabidopsis cultured cells. Plant J. 31, 499-515. doi: 10.1046/j.1365-313X.2002.01372.x

Valkonen, M., Ward, M., Wang, H., Penttilä, M., and Saloheimo, M. (2003). Improvement of foreign-protein production in Aspergillus niger var. awamori by constitutive induction of the unfolded-protein response. Appl. Environ. Microbiol. 69, 6979-6986. doi: 10.1128/AEM.69.12.6979-6986.2003

van Gemeren, I. A., Beijersbergen, A., van den Hondel, C. A., and Verrips, C. T. (1998). Expression and secretion of defined cutinase variants by Aspergillus awamori. Appl. Environ. Microbiol. 64, 2794-2799.

van Munster, J. M., Nitsche, B. M., Krijgsheld, P., van Wijk, A., Dijkhuizen, L., Wösten, H. A., et al. (2013). Chitinases CtcB and CfcI modify the cell wall in sporulating aerial mycelium of Aspergillus niger. Microbiology 159, 1853-1867. doi: 10.1099/mic.0.067967-0

Wanka, F., Cairns, T., Boecker, S., Berens, C., Happel, A., Zheng, X., et al. (2016). Tet-on, or Tet-off, that is the question: advanced conditional gene expression in Aspergillus. Fungal Genet. Biol. 89, 72-83. doi: 10.1016/j.fgb.2015. 11.003
Weaver, P. L., Sun, C., and Chang, T. H. (1997). Dbp3p, a putative RNA helicase in Saccharomyces cerevisiae, is required for efficient pre-rRNA processing predominantly at site A3. Mol. Cell. Biol. 17, 1354-1365. doi: 10.1128/MCB.17.3.1354

Wiebe, M. G., Karandikar, A., Robson, G. D., Trinci, A. P., Candia, J. L., Trappe, S., et al. (2001). Production of tissue plasminogen activator (t-PA) in Aspergillus niger. Biotechnol. Bioeng. 76, 164-174. doi: 10.1002/bit.1156

Winzeler, E. A., Shoemaker, D. D., Astromoff, A., Liang, H., Anderson, K., Andre, B., et al. (1999). Functional characterization of the S. cerevisiae genome by gene deletion and parallel analysis. Science 285, 901-906.

Wright, J., Kahn, R. A., and Sztul, E. (2014). Regulating the large Sec7 ARF guanine nucleotide exchange factors: the when, where and how of activation. Cell. Mol. Life Sci. 71, 3419-3438. doi: 10.1007/s00018-014-1602-7

Wucherpfennig, T., Lakowitz, A., Driouch, H., Krull, R., and Wittmann, C. (2012). Customization of Aspergillus niger morphology through addition of talc micro particles. J. Vis. Exp. e4023. doi: 10.3791/4023

Zhang, C. J., Cavenagh, M. M., and Kahn, R. A. (1998). A family of Arf effectors defined as suppressors of the loss of Arf function in the yeast Saccharomyces cerevisiae. J. Biol. Chem. 273, 19792-19796. doi: 10.1074/jbc.273.31.19792

Conflict of Interest Statement: The authors declare that the research was conducted in the absence of any commercial or financial relationships that could be construed as a potential conflict of interest.

Copyright (C) 2018 Fiedler, Cairns, Koch, Kubisch and Meyer. This is an open-access article distributed under the terms of the Creative Commons Attribution License (CC $B Y)$. The use, distribution or reproduction in other forums is permitted, provided the original author(s) and the copyright owner are credited and that the original publication in this journal is cited, in accordance with accepted academic practice. No use, distribution or reproduction is permitted which does not comply with these terms. 